\title{
Rapid isolation and resolution immune evasion and viral fitness across contemporary SARS-CoV-2 variants
}

\section{Anupriya Aggarwal}

UNSW Sydney

\section{Alberto Stella}

Kirby Institute UNSW

\section{Anouschka Akerman}

Kirby Institute UNSW

\section{Gregory Walker}

Serology and Virology Division (SAViD), NSW Health Pathology, Randwick, Australia.

\section{Vanessa Milogiannakis}

Kirby Institute UNSW

\section{Samantha McAllery}

Kirby Institute UNSW

\section{Camille Esneau}

Hunter Medical Research Institute, University of Newcastle

\section{Mariana Ruiz Silva}

Kirby Institute UNSW Sydney

\section{Yonghui Lu}

Serology and Virology Division (SAViD), NSW Health Pathology, Randwick

\section{Charles Forster}

Serology and Virology Division (SAViD), NSW HP SEALS, Randwick, Australia.

\section{Sebastiaan van Hal}

Royal Prince Alfred Hospital https://orcid.org/0000-0001-5852-4155

\section{Vennila Mathivanan}

Kirby Institute UNSW

\section{Christina Fitcher}

Kirby Institute UNSW

\section{Alexandra Carey Hoppe}

Kirby Institute UNSW

\section{Mee Ling Munier}

Kirby Institute UNSW Sydney https://orcid.org/0000-0002-6419-142X

\section{David Darley}


St Vincent's Hospital, Sydney https://orcid.org/0000-0002-9721-101X

\section{Gail Matthews}

St Vincent's Hospital, Sydney

\section{Damien Starck}

St Vincent's Hospital, Sydney, New South Wales, Australia.

\section{Roselle Robosa}

St Vincent's Hospital, Sydney, New South Wales, Australia. https://orcid.org/0000-0002-7886-8219

\section{Nathan Bartlett}

Hunter Medical Research Institute, University of Newcastle

\section{William Rawlinson}

Prince of Wales Hospital

\section{Anthony Kelleher}

Kirby Institute UNSW

\section{Stuart Turville ( $\nabla$ sturville@kirby.unsw.edu.au )}

Kirby Institute UNSW Sydney https://orcid.org/0000-0003-1918-5343

\section{Article}

\section{Keywords:}

Posted Date: January 13th, 2022

DOI: https://doi.org/10.21203/rs.3.rs-1210846/v1

License: (c) (i) This work is licensed under a Creative Commons Attribution 4.0 International License. Read Full License

Version of Record: A version of this preprint was published at Nature Microbiology on May 30th, 2022. See the published version at https://doi.org/10.1038/s41564-022-01135-7. 
Rapid isolation and resolution of immune evasion and viral fitness across contemporary SARS-CoV-2 variants

Anupriya Aggarwal ${ }^{1 *}$, Alberto Ospina Stella ${ }^{1 *}$, Gregory Walker ${ }^{3}$, Anouschka Akerman ${ }^{1}$, Vanessa Milogiannakis ${ }^{1}$, Samantha McAllery ${ }^{1}$, Camille Esneau ${ }^{2}$, Mariana Ruiz Silva ${ }^{1}$, Yonghui Lu ${ }^{3}$, Charles Forster ${ }^{3}$, Sabastiaan Van $\mathrm{Hal}^{4}$, Vennila Mathivanan ${ }^{1}$, Christina Fichter ${ }^{1}$, Alexandra Carey Hoppe ${ }^{1}$, Mee Ling Munier ${ }^{1}$, David Darley ${ }^{5}$, Gail Matthews ${ }^{1,5}$, Damien John Starck $^{6}$, Roselle Robosa ${ }^{6}$, Nathan Bartlett ${ }^{2}$, William Rawlinson ${ }^{3}$, Anthony D Kelleher ${ }^{1,5}$, Stuart Turville $^{1}$

${ }^{1}$ The Kirby Institute, University of New South Wales, New South Wales, Australia.

${ }^{2}$ Hunter Medical Research Institute, University of Newcastle.

${ }^{3}$ Serology and Virology Division (SAViD), NSW HP SEALS, Randwick, Australia.

${ }^{4}$ Royal Prince Alfred Hospital, New South Wales, Australia.

${ }^{5}$ St Vincent's Hospital, Sydney, New South Wales, Australia.

${ }^{6}$ Molecular Diagnostic Medicine Laboratory, Sydpath, St Vincent's Hospital, Sydney, New

South Wales, Australia.

Corresponding Author:

Stuart G. Turville

The Kirby Institute, UNSW Australia

Office 529 Level 5 Wallace Wurth Building,

UNSW, Sydney NSW 2052

Telephone: +61 (02) 93850462

Email: sturville@kirby.unsw.edu.au

${ }^{1}$ The Kirby Institute, University of New South Wales, New South Wales, Australia.

*Equal contribution 


\begin{abstract}
From late 2020 the world observed the rapid emergence of many distinct SARS-CoV-2 variants. At the same time, pandemic responses coalesced into significant global vaccine rollout that have now significantly lowered COVID-19 hospitalisation and mortality rates in the developed world. Over this period, we developed a rapid platform (R-20) for viral isolation and characterisation using primary remnant diagnostic swabs. This combined with quarantine testing and genomics surveillance, enabled the rapid isolation and characterisation of all major SARS-CoV-2 variants (all variants of concern (VOC) and six variants of interest (VUI)) globally within a four-month period. This platform facilitated viral variant isolation and enabled rapid resolution of variant phenotype by determining end-point viral titre from primary nasopharyngeal swabs and through ranking of evasion of neutralising antibodies. In late 2021, when the Delta variant was dominating, Omicron rapidly emerged. Using this platform, we isolated and tested the first cases of this variant within Australia. In this setting we observed Omicron to diverge from other variants at two levels: Firstly, it ranks as the most evasive to neutralisation antibodies compared to all VOCs and major VUIs. Secondly, it no longer engages TMPRSS2 during late stages of fusion.
\end{abstract}




\section{Introduction}

As of December 2021, severe acute respiratory syndrome coronavirus 2 (SARS-CoV-2), the causative agent of COVID-19, has accounted for more than 250 million infections and more than 5 million deaths worldwide (https://covid19.who.int/). Within the first 12 months of the pandemic the virus accumulated polymorphisms primarily within its Spike glycoprotein that have initially driven higher levels of transmission ${ }^{1-4}$ and significantly reduced efficacy of vaccines in preventing symptomatic disease particularly with the most immune evasive Variant of Concern (VOC) Beta 5,6. In 2021, SARS-CoV-2 accelerated its spread initially in India through the VOC Delta and this now has become the dominant variant globally, supplanting Alpha, Beta and Gamma (https://nextstrain.org/ncov/open/global). Biological mechanisms for the fitness dominance of Delta include its increased Angiotensin Converting Enzyme 2 (ACE2) affinity through 452 and 478 Spike polymorphisms ${ }^{4}$, combined with the furin cleavage site polymorphism P681R that drives more efficient late-stage viral fusion in concert with the serine protease TMPRSS $2^{7}$. Whilst these observations are in vitro, high viral loads sustained within the nasopharyngeal tract ${ }^{8}$, a significantly higher secondary attack rate compared to the previous dominating VOC Alpha ${ }^{9}$ and other viral variants, all suggest that these changes have significant implications on in vivo fitness. Whilst vaccines were highly efficacious against Delta, the rapid emergence of the Omicron variant in late 2021 has led to high transmission rates in both convalescent and vaccinated groups.

During 2021 our aim was to develop a simple and rapid means of viral variant isolation and characterisation. To do so, we developed a series of platforms that combined firstly fast turnaround of whole genome sequencing ${ }^{10}$, secondly rapid and sensitive viral isolation and finally rapid viral characterisation. The utility of our platforms was then tested stringently at several levels. Firstly, in rapid isolation of many globally relevant variants isolated from swabs with low viral loads (PCR Ct $>30$ ). Secondly, we characterised variants against a range of humoral responses and therapeutics and established relative viral fitness, quantification and characterisation to enable studies of neutralisation (or equivalent therapeutic screens) within one week of obtaining the original nasopharyngeal swab. Finally, with the recent expansion of Delta in the Australian community, we demonstrate the utility of our methods to rapidly and sensitively determine in vivo viral titres directly from nasopharyngeal swabs overnight. When the latter values are combined with diagnostic PCR, it enables real-time in vivo measurement of infectivity to particle ratio calculations (viral fitness). Following characterisation of our platform, Omicron was observed in vaccinated travellers returning to Australia in late November. In this latter setting, we rapidly isolated and tested Omicron for humoral neutralisation within one week of receiving the swab specimens and provided the first evidence of patient infectivity when Omicron was present initially in many breakthrough vaccine cases. The latter highlighted the solutions herein are valuable for the continued COVID-19 response as they enable the development of a scale of relative variant threat that combines measures of viral fitness with measurements of humoral/therapeutic evasion. Importantly, this platform can be performed at scale and in formats consistent with global accessibility and use. 


\section{Results}

\section{Rapid isolation of all VOCs and major VUIs through Australian quarantine}

In late 2020 and the first half of 2021, NSW hotel quarantine accommodated approximately half of all people entering Australia with less than $1 \%$ positive for SARS-CoV-2 infection (https://www.health.gov.au/sites/default/files/documents/2020/10/national-review-of-hotelquarantine.pdf). As part of the COVID-19 response, rapid molecular surveillance was established with $3^{\text {rd }}$ generation single molecule sequencing technologies ${ }^{10}$, enabling greater than $80 \%$ of all positive cases to be sequenced. As sequencing was carried out, nasopharyngeal swabs were stored with variable cold chains, ranging from cryopreservation within 24 hours at $-80^{\circ} \mathrm{C}$ to prolonged periods of time at $4^{\circ} \mathrm{C}$, followed by storage at $-80^{\circ} \mathrm{C}$. Initial attempts to recover viruses from swabs were not very successful using VeroE6 cells, with less than 1 in 5 swabs culture positive.

In this setting we introduced a hyper-permissive HEK239T-based cell line that co-expresses ACE2 and TMPRSS2 (HAT-24) that was 100 to 1000-fold greater in sensitivity than the VeroE6 cell line (Supplementary Figure 1). Sensitivity was based not only on the expression of physiologically relevant receptors ACE2 and TMPRSS2 ${ }^{11}$, but the selection of a clone that lacked viral restrictions as revealed by significant dose-dependent cytopathic effect (CPE) accumulating after 8 hours post infection (Movie S1). Within the HEK293T line, innate viral immunity, specifically TAg and adenovirus E1A expression, in the parental HEK293T line antagonise antiviral responses by countering IRF3 or IFN-dependent transcription downstream of RNA and DNA sensor activation ${ }^{12-16}$. Whilst we identified and tested many HEK293T clones expressing ACE2 and TMPRSS2 (See Supplementary Figure 1A-F for a representative clone of 24 (HAT-10 versus HAT-24 in 6 contemporary variants)), there was only one clone that was hyper-permissive to infection. In this latter setting we hypothesised that the abovementioned lack of viral restrictions in the HEK293T line are augmented in this specific cellular clone and as such enables rapid viral infection and spread culminating in the rapid appearance of unequivocal CPE that can be scored using automated cell counts, viability assays and/or visual scoring using simple inverted tissue culture microscopes.

The introduction of the hyper-permissive line HAT-24 enabled virus isolation from greater than $80 \%$ of PCR-positive swabs, with all VOCs (Alpha, Beta, Gamma and Delta) and 6 VUIs (Kappa, Eta, Zeta, Epsilon, Iota and Lambda) isolated successfully over a four-month period in early 2021 (Figure 1A). Visual scoring of cultures revealed many were positive for CPE after over-night culture, with a significant correlation $(r=-0.88 ; \mathrm{p}=0.02)$ of average diagnostic PCR Ct values and titres (50\% Tissue Culture Infectious Dose; TCID50/ml) (Figure 1B). Expansion of high titre cultures within this line was limited by the appearance of rapid CPE with peak viral titres at approximately $10^{3} \mathrm{TCID} 50 / \mathrm{ml}$. Given the rapid appearance of CPE overnight (see Figure 1C uninfected versus Figure 1D-E overnight infection and 48 hours post infection with primary swab, respectively), we reasoned this was the rate limiting step in generating higher viral titres. To enable the latter, simple co-culture of the infected HAT-24 cell line 2 to 3 days post infection (when the culture has succumbed to maximal CPE) with the VeroE6-TMPRSS2 cell line to enable high titre stocks to be generated within 1 to 2 days. Across all swabs, high titre viral stocks were obtained within 4 to 5 days following receipt of the primary swab.

\section{R-20: A rapid overnight- high content assay for rapid screens using live SARS-CoV-2}

Initial use of the HAT-24 line revealed that even a small number of infectious virions could lead to extensive CPE after 72 hours. However, from rapid isolation of variants, we observed 
extensive CPE accumulated rapidly following short term culture in primary samples with PCR $\mathrm{Ct}$ values lower than 20 (Figure 1C-E). As we observed CPE in the HAT-24 line shortly postinfection, our next aim was to determine if this approach could be used to rapidly determine viral titres and screen serum/therapeutics for anti-viral activity in a high content setting.

For initial comparisons we tittered virus using the HAT-24 line and VeroE6 cultures over 3 days to establish end-point titres as TCID50 using the Spearman-Karber method ${ }^{17}$. In parallel, we also measured dose-dependent loss of $50 \%$ of nuclei (50\% Lethal Infectious Dose (LD50)) through live cell staining with Hoechst 3342 combined with high content machine driven scoring and counting using both the VeroE6 and HAT-24 cell lines in two formats; after 20 hours and after 3 days of culture. Analysis for the 20-hour truncated format was only performed using the HAT-24 line, as the VeroE6 line did not result in sufficient CPE after 20 hours of culture (Figure 2A). Subsequent testing using the VeroE6-TMPRSS2 line ${ }^{18}$ also revealed similar lack of CPE after 20 hours of culture. High content microscopy of the HAT-24 line 20 hours post-infection revealed dose-dependent loss of cells as revealed in sigmoidal dosedependent curves with only minor variation between technical replicates (Figure 2B-C). Whilst sigmoidal curves could be fitted to VeroE6 cultures after 3 days, it was apparent this longer culture period amplified variation between replicates (Figure 2B-C).

End-point titres using the Spearman-Karber method were calculated at approximately $0.5 \times 10^{6}$ TCID50/ml in VeroE6 and $0.5 \times 10^{8}$ TCID50/ml in the HAT-24 across all 12 variants tested (Figure 2D-E). Importantly across all variants, the titres in HAT-24 were within a five-fold range, highlighting the overnight titres were from restricted rounds of infection and therefore did not augment any viral fitness gains. In the VeroE6 line, we observed earlier clades from April 2020 sustained higher titres than contemporary clades isolated in early 2021 (Figure 2DE). Given the linearity of the dose-dependent curves, for stringency we established titres based on $50 \%$ loss of nuclei (LD50) ${ }^{19}$. This was then formally compared with traditional TCID50 assays after 3 days culture using the Spearman-Karber method. Whilst the magnitude of the LD50 dilution values at 24 hours was lower than that calculated by the Spearman-Karber method at 72 hours, there was significant correlation to LD50 values (Spearman coefficient of correlation, $r=0.62, \mathrm{p}<0.05$ ) across all variant titres tested. We subsequently will refer to the overnight method in HAT-24 as R-20, a rapid 20-hour full virus screening platform.

\section{The R-20 platform enables rapid, accessible and scalable screening}

The VeroE6 line serves as the current major cell line utilised worldwide for screening of humoral immunity and/or immunotherapeutics to SARS-CoV-2 in the form of monoclonal antibodies or polyclonal clinical products like plasma derived hyper-immune IgG. Therefore, we cross-validated the R-20 platform against the longer VeroE6 assay ${ }^{\mathbf{2 0}}$. For initial cross validation we used the Ab-3467 monoclonal antibody, as it recognises a conserved epitope within SARS-CoV-2 spike, and thus is active against a broad range of SARS-CoV-2 variants 21. All cross-comparisons between the R-20 platform and the VeroE6 line were carried out using 12 primary low passage SARS-CoV-2 isolates expanded over a 4-day period under identical conditions (see methods). This included two early clades (A.2.2 and B1 (B.1.319)), four VOCs (Alpha, Beta, Gamma and Delta) and the majority of VUIs (Epsilon, Eta, Zeta, C36 and Lambda). In the setting of Ab-3467, all variants were neutralised within a tight range of IC50s within the R-20 assay (Figure 3A-C). In comparison, with VeroE6s we resolved potencies over a larger range but closely correlated with the R-20 assay $(r=0.94 ; \mathrm{p}<0.001)$ (Figure 3A-C). This greater variation in IC50, alongside the larger standard deviations observed with the VeroE6 cell line, is consistent with viral replication over several days 
compared to the restricted rounds of infection observed in the R-20 assay (i.e. First round cellfree viral entry and second round cell-cell spread that reveals dose-dependent CPE).

To summarise the results, the R-20 assay correlated well with the longer duration VeroE6 format. The key difference in the R-20 assay was overnight acquisition that results in observations with very low technical replicate variability, excellent dose-dependent linearity with resolution of 12 primary SARS-CoV-2 variants including all VOCs. Whilst in these experiments the R-20 system was performed using high content microscopy, we demonstrated linearity of the R-20 assay using an inexpensive viability dye (Resazurin; Alamar Blue) and standard optical multi-titre plate reader set to reading absorbance values at 570 and $600 \mathrm{~nm}$ (Supplementary Figure S2 A-B). The use with the latter increases the accessibility to the platform by reducing the need for a high content microscope.

Assessment of immune evasion of SARS-CoV-2 at peak humoral responses in convalescent and BNT162b2-vaccinated donors

Having demonstrated the advantages of the R-20 platform in these initial comparisons, we turned to ranking of variants across a panel of sera from convalescent donors $(n=24)$ and mRNA vaccine (BNT162b2) recipients $(n=24)$. In late 2020, Spike polymorphisms associated with fitness gains (e.g. N501Y, L452R, P681R/H) emerged alongside those associated with humoral evasion (e.g. E484K). This led to concerns that many variants could effectively evade existing convalescent or vaccine-induced responses and enable re-infection. As we isolated all variants across a relatively short time period, each was expanded using the same cell lines and used with the same low passage number.

Across individual donors, we did observe a range of responses to variants in convalescent versus BNT162b2 vaccine responses (Figure 4A-D). For instance, across all vaccine recipients, breadth was similar across all donors tested, with Beta being the most evasive (Supplementary Table S1, S3, S5). In contrast the panel of convalescent donors derived from the ADAPT cohort highlighted distinctly different phenotypes (Supplementary Table S2, S4 and S6.). For instance, across the 24 ADAPT donors, the five donor sera that produced end point titres $>1 / 80$ to Beta (AD007, AD008, AD010, AD043), all maintained titres across all variants tested at potencies equal to or greater than Beta. Donor AD062 was an exception, as this volunteer had a good response to early clade variants but in contrast did not reach end point titres to the variants Epsilon, Delta, Kappa, Lambda, Gamma and Beta (Supplementary Table S2 and Supplementary Figure S3). With the exception of Gamma and Beta, all of these variants shared the L452R or L452Q polymorphism in the Spike receptor-binding domain (RBD). In contrast, two donors (AD010 and AD016; Supplementary Figure S3), were observed to maintain high titres and breadth across all variants, with less than a 2-fold reduction in titre from the early A.2.2 clade to any of the 11 variants tested. Both responses were superior in breadth to any vaccinated donor tested.

To rank variants based on humoral evasion across all donors, we determined average fold reductions across both the BNT162b2 and convalescent cohorts in the R-20 and VeroE6 assays. Consistent with previous studies, we found Beta to be the most immune-evasive among all the variants studied in both vaccine and convalescent samples across both platforms (Figure 4G-J) ${ }^{22-26}$. Across the two platforms, there was concordance with Lambda and Kappa having the next greatest reduction in neutralisation titres. Overall, the fold reductions in the convalescent panel were approximately half of that observed in BNT162b2 vaccinated donors. Although it must 
be noted that this selected subset of the ADAPT cohort were enriched for donors with high neutralisation responses.

Rapid resolution of in vivo infectivity and fitness of variants circulating in the community From the isolation of 12 viral variants, we established that viral end-point titres using the R-20 platform were initially correlated with diagnostic PCR (Ct values). However, each sample had a variable cold-chain that could have influenced the result. To determine the utility of the R20 assay for determining viral fitness from ex vivo samples, we curated two sets of specimens with the same cold chain storage that were collected in the community as part of routine diagnostic testing. All samples were collected primarily during the acute phase of infection. In brief, all nasopharyngeal swabs in Australia undergo viral genomic sequencing as part of the COVID-19 response and tracing. As part of the diagnostic setting at St Vincent's Hospital, positive swabs are set aside within 24 hours of collection and aliquots frozen at $-80^{\circ} \mathrm{C}$ for cold chain shipment for later sequencing analysis and aliquots biobanked for later testing if required. At the end of 2020 a quarantine breach led to singular seeding and community spread of the B.1.517.1 clade that only bears the D614G and N501T polymorphism within Spike. Whilst this clade is not common worldwide, it did provide primary samples that had the same genotype that was closely related to early circulating B clade variants with only the D614G spike change. At that time, the Australian community was COVID-19 vaccine naïve. The second cohort was derived during the month of July 2021 in the early stages of the recent Australian Delta outbreak. At that time $99 \%$ of cases in NSW were transmissions in the unvaccinated and thus representative of samples from a vaccine naïve population (https://www.health.nsw.gov.au/Infectious/covid-19/Documents/covid-surveillance-report-

20210813.pdf). Whilst using R-20 we could rapidly identify those individuals with high infectious viral loads, we also used the longer culture period for increased sensitivity in addition to enabling increased stringency through two rounds of viral titre scoring (Figure 5AH). Using this strategy, we observed sensitivity of 20-hour versus 96-hour cultures to be similar and both were observed to have similarly significant correlations with average $\mathrm{Ct}$ values $(r=-$ $0.8585, \mathrm{p}<0.0001$ and $r=-0.8606, \mathrm{p}<0.0001$, respectively). At both time points virus could be detected at $\mathrm{Ct}$ values equal to and greater than 37 (Figure 5H). In comparison, recent studies using similarly collected and stored samples and the VeroE6-TMPRSS2 line, observed limits of detection at $\mathrm{Ct}$ values around 25 (https://www.medrxiv.org/content/10.1101/2021.09.07.21263229v1.full.pdf). For this study, we could not formally compare the HAT-24 line with either the VeroE6 and/or VeroE6TMPRSS 2 due to the limiting volume of primary swab material (100 $\mu 1$ in total). However, using expanded cultured virus, we observed relative rank sensitivities of viral titres HAT24>VeroE6-TMPRSS2>VeroE6, with each increase in sensitivity being greater than an order of magnitude.

Importantly, using the HAT-24 cells in the R-20 format, we determined many Delta primary swabs with diagnostic PCR Ct values of less than 20 to have titres similar to that observed in laboratory expanded cultures and that these differences could be easily resolved following overnight culture (Figure 5A-H). We then generated lines of linear regression for each variant and observed resolution of Delta versus the B.1.517.1 as a upward shift in the linear regression for the former variant (Figure 5I). Using this linear regression, we calculated Delta to be 9.1fold more infectious virus than the B.1.517.1 strain with similar genotype to early circulating variants, for samples with the same amount of total viral RNA ( $\mathrm{p}=0.0004)$. Given Delta is presently the major circulating variant globally, we then compared titres across age groups and observed no significant correlation $(r=0.10556 ; \mathrm{p}=0.3674)$, with titres in several children under 12 years of age as high as that observed in adults (Figure $5 \mathrm{~J} ;>10^{7}$ TCID50/ml). To 
conclude, this analysis confirmed primary swabs can be used to calculate end point titres and then combined with diagnostic PCR values to rapidly determine relative viral fitness. The primary limitation to the latter fitness analysis is the requirement of significant community spread of virus and collection of primary swabs to determine the relative fitness of emerging contemporary variants. To address this latter concept with the R-20 platform, we investigated the relative fitness of Alpha, Delta and Lambda, as Delta has circulated and supplanted both Alpha and Lambda globally. As the closest surrogate for in vivo expansion, we infected primary ALI cultures 27 with the same multiplicity of infection using culture expanded viral preparations. Replication in primary cultures would then reveal greater fitness of each isolate upon testing the titres in an identical manner to that used in patient nasopharyngeal swabs. Using this approach, end-point titres from primary ALI cultures 3 days post-infection were observed to resolve the fitness of each variant with Delta $>$ Lambda $>$ Alpha (Figure $6 \mathrm{~K}$ ). This rank of variant fitness in vitro is consistent with that known of Delta's versus Alpha and Lambda's fitness in the community ${ }^{28}$. To conclude, this latter approach could be used when the frequency of emerging variants is low and then used in combination with in vivo fitness scores using end-point titres of primary swabs.

\section{Rapid isolation and characterisation of the first Omicron case within Australia}

The first case of Omicron in Australia was detected on the $27^{\text {th }}$ of November in two vaccinated, asymptomatic returning travellers from South Africa. Upon receipt of the primary nasopharyngeal swab, virus was expanded within the HAT-24 within 48 hours and then further expanded using the VeroE6 cell line. Whilst Omicron shares many RBD polymorphisms with other VOCs, it is immediately apparent that it has significantly diverged from all previous variants that appeared in 2021 (Figure 6A-B.). As observed in previous analysis of individual donors, it was apparent that in both vaccinated and convalescent responses there was a continuum of responses that influenced the fold change in the response. Rather than test more donors on an individual basis, we utilised polyclonal preparations generated either from pools of thousands of convalescent plasma donors or harvesting of thousands of plasma donors at a time when vaccination was at its peak in the USA (donors were based in the USA). This approach then would give a broad snapshot of a variant's evasiveness at the population level. Whilst titres were lower than other variants in the HAT-24 line, CPE still accumulated overnight with extensive syncytia produced (Figure 6C). Comparison across all variants revealed Omicron as the most evasive variant, with the highest fold reductions to both vaccine and convalescent pooled serum 4 times greater than that observed with Beta (Figure 6D-E).

Given the rapid spread of Omicron in the community, we were able to access many primary nasopharyngeal swabs to test for infectivity per diagnostic $\mathrm{Ct}$ value. At the time of Omicron spread within the community, the population of NSW was over $90 \%$ vaccinated with two doses of either BNT162b2 or ChAdOx1 nCoV-19. In testing of over 35 Omicron primary samples, we observed a significant downward shift in the linear regression compared to Delta (Figure $6 \mathrm{~F}$ ). Whilst vaccination could influence this downward shift, the rapid spread in the community contrasted the observation that this variant was less infectious per particle compared to early SARS-CoV-2 variants. Given the slower growth observed in the VeroE6-TMPRSS2 line, we tested Omicron versus Delta for its ability to be blocked by the TMPRSS2 inhibitor Nafamostat using the HAT-24 line. In contrast to Delta, we observed Omicron to be refractory to Nafamostat across all concentrations tested (Figure 6G). Therefore, we conclude the cellular entry pathway for Omicron has diverged from all variants prior to Delta and throughout the pandemic. Given TMPRSS2 significantly increases SARS-CoV-2 entry for other variants (especially Delta), the downward shift in the linear regression would be expected with Omicron using the HAT-24 cell line. Given the increased transmissibility of Omicron within the 
community, the use of other proteases and/or cathepsins that are present in respiratory tract may have resulted in a change in cellular tropism. Thus, comparisons of variants proceeding Omicron must be framed in the context that the entry pathway has now significantly diverged. To understand this tropism further, entry requirements will need to be mapped for Omicron.

\section{$\underline{\text { Discussion }}$}

Herein our aim was to enable platforms to accelerate the COVID-19 response at two principal levels. Firstly, rapid, sensitive and faithful isolation of primary isolates of SARS-CoV-2 at even low viral loads. Secondly, enable rapid characterisation of their relative threat. The latter ranged from the ability to rapidly determine the evasion potential against a humoral response and/or therapeutics through to assessment of viral fitness in vivo and in vitro. For rapidly spreading pandemics like COVID-19, the time taken for stringent resolution of a viral phenotype often lags and we often retrospectively determine phenotypes as a variant is spreading throughout the community. Although as regions and entire countries manage surging cases of COVID-19, early warning systems that are evidence-based are the best ways to inform policies regarding the threat of a variant if it were to establish community spread. Time and precision is paramount in this context and the platform established herein enables this at several levels, from time to virus isolation and expansion through to characterisation.

Contemporary variants that have dominated in 2021 all share similar changes within their Spike glycoprotein ${ }^{1-4}$. The changes are related to humoral evasion and viral fitness gains that have significantly increased transmissibility in variants like Delta. Thus, we will discuss the rapid resolution of primary viral phenotypes at two levels. Firstly, with respect to evasion of the convalescent and vaccine-induced humoral response and secondly with respect to viral fitness gains. The ability of contemporary variants to evade a humoral response has recently received significant attention as it primarily informs if the current vaccine formulations based on earlier circulating clades are still sufficient in mediating appropriate breadth to emerging variants and/or if updated booster formulations are needed. Although observations of fold resistance to vaccines responses have varied significantly across studies, from observations of significant humoral evasion in some studies through to more conservative observations in others ${ }^{4,22,29}$. In this setting a contemporary variant's threat in evading an initial humoral response can be conflicting and only fully resolves when efficacy of a vaccine response is observed during substantial community spread. Although the discordance in results is multifactorial, a key underlying issue is the differential fitness of each variant that can be compounded by several rounds of viral replication over several days in live cell assays. Herein by limiting the rounds of viral replication in a simplified assay, we observe conservative fold reductions in in vitro vaccine efficacy consistent with that outlined by Planas et al and Lui et al ${ }^{22,29}$. The importance of the latter two studies is the control for viral fitness either by time or through assay read-outs that restrict fitness in plaque assays by increasing media viscosity. Longer culture conditions using a 3-day read-out, not only significantly amplified variation in technical replications, but also increased fold-reductions to humoral responses by 2 to 3 -fold. Importantly, conservative fold reductions in sensitivity to neutralising antibodies demonstrated herein using the R-20 platform are also consistent with that observed in many large-vaccinated cohorts and studies. Primarily as Beta has routinely be observed across many different vaccines to be the most evasive variant ${ }^{5,6}$ (https://doi.org/10.1101/2021.09.27.21264163), whilst reductions in vaccine efficacy to Alpha and Delta have been moderate ${ }^{30}$. Whilst Beta was initially the most evasive variant, the emergence of Omicron in November 2021 has revealed a significantly evasive and highly transmissible variant. Using our platform herein, tested across many variants that appeared in 2021, we could rapidly capture and rank Omicron in its ability to be neutralised by 
either the vaccine or convalescent response at the community level by the use of large pools of thousands of plasma donors that represent clinical polyclonal IgG. Whilst at the time of submission of this work for review, many laboratories had submitted pre-prints on the relative fold reduction of neutralisation titres of Omicron in both convalescent and vaccinated populations. As observed with Delta, the fold reductions covered a significant range. Importantly, we now need to determine the vaccine efficacy in areas of Omicron community spread and in doing so align each in vitro assay with what is observed in vivo. In this latter context, our assays combined with modelling observe a reduction of two dose vaccine efficacy to $37.2 \%$ \{https://www.medrxiv.org/content/10.1101/2021.12.14.21267772v1 $\}$ and is consistent with the recent observation of vaccine efficacy observed in South Africa at 33\% (https://www.discovery.co.za/corporate/health-insights-omicron-outbreak-analysis).

Secondly, in turning our attention to fitness gains, whilst initially increased receptor affinity for ACE2 was observed in all VOCs by changes within the RBD, it was further changes in the furin cleavage site that enabled a fitness advantage over other VOCs through increased viral fusion when TMPRSS2 was present ${ }^{31}$. Thus, observations of potential increases in fitness and transmissibility need to address that observed with in vivo entry and subsequent amplification. ACE2 and TMPRSS2 are the relevant in vivo entry pathway ${ }^{32}$ and expression in the R-20 platform is one of the foundations of its sensitivity and utility for isolation of emerging contemporary viral variants. Whilst isolation, expansion and rapid dissection of each variant is of immense importance, even more so is how the variant is replicating in vivo. Whilst much attention has been placed on the increasing viral loads associated with Delta in either unvaccinated or breakthrough infection ${ }^{33-35}$, this does not inform on the real risk of an individual's ability to spread the virus or how previous vaccination may influence this (i.e. their actual infectious viral load and not their viral load based on nucleic acid detection). Through rapid end-point titres, we could observe this infectious potential in an individual, and we could also determine the relative increase in infectivity per viral load between variants. Importantly, moving forward, rapid enumeration of infectivity of swab samples in breakthrough infections of vaccinated communities and their correlation to viral neutralisation responses will further inform vaccine efforts. In efforts to curb the pandemic trajectory, it will be important to carry out studies on future vaccines (booster or otherwise) and related formulations and their impact on the infectivity of vaccine recipients that may be infected.

SARS-CoV-2 variants prior to Omicron could be ranked against each other in the analyses listed above. That said, when variants change their mode of entry (i.e. no longer utilise TMPRSS2), relative viral fitness can no longer be compared across previous variants. This was readily observed in a downward shift in the linear regression of infectivity per $\mathrm{Ct}$ value for Omicron. This shift is not consistent with the rapid spread of Omicron, which globally has proved to accelerate spread within the community far more than what we have observed with other variants. Rather, this simply highlights that the requirements for Omicron viral entry have changed and could no longer benefit from the co-expression of ACE2 and TMPRSS2 as was observed in all variants prior. To increase the sensitivities of assays herein and assays elsewhere we will need to now incorporate the requirements that Omicron uses in vivo. Preliminary studies have observed a lowering in ability of Omicron to enter cell lines Caco2 and Calu3 (derived from colon and lung, respectively) and primary lung cultures versus an increase in its ability to enter cells derived from bronchial samples (https://doi.org/10.1016/j.cell.2021.12.032). If the latter change in tropism is reflected in vivo, a molecular understanding of how this has proceeded via a change in cellular entry will be required to ensure we can continue to update platforms described herein. If entry and tropism of Omicron has significantly diverged, the pathogenesis of the virus may have diverged as well. 
Recent reports support a significant shift in tropism from the lower lung to the upper bronchial tissue (https://www.med.hku.hk/en/news/press/20211215-omicron-sars-cov-2-infection). Whilst this is consistent with increased transmissibility, it is still unclear if this change in tropism has increased or decreased disease severity. That said, moving forward we will now need to consider two distinct pools of SARS-CoV-2 viral variants and the potential emergence thereof. The first pool of variants with an entry phenotype of variants pre-Omicron and defined by the use of ACE2 and TMPRSS2. The second pool defined by variants with a similar tropism and entry pathway to Omicron. In addition to this, we must also be prepared to track variants that may switch between these entry pathways or utilise both. Indeed, there are precedents for other RNA viruses to both mix or switch tropism ${ }^{36}$.

To conclude, the research herein provides an accessible, easy to use pipeline that enables resolution of SARS-CoV-2 variant threat at several important levels: Firstly, in its ability to sensitively combine viral isolation, expansion and characterisation of primary viral isolates from nasopharyngeal within one week. Secondly, in its ability to enable screening of thousands of compounds/samples reproducibly and efficiently. Whilst this was used in the resolution of the key phenotypes of many contemporary SARS-CoV-2 variants that emerged in 2021, its utility was tested with the emergence of Omicron at the conclusion of this study. If the SARSCoV-2 pandemic will now be based on variants based on Omicron, we will need to rapidly resolve the entry pathway to enable screening of relevant entry inhibitors. Alternately, if a variant would appear to outcompete Omicron that uses the ACE2-TMPRSS2 pathway, the assays herein and those developed for Omicron, can readily be mobilised to resolve the appearance of SARS-CoV-2 variants with differing tropism. 


\section{Figures}

Figure 1
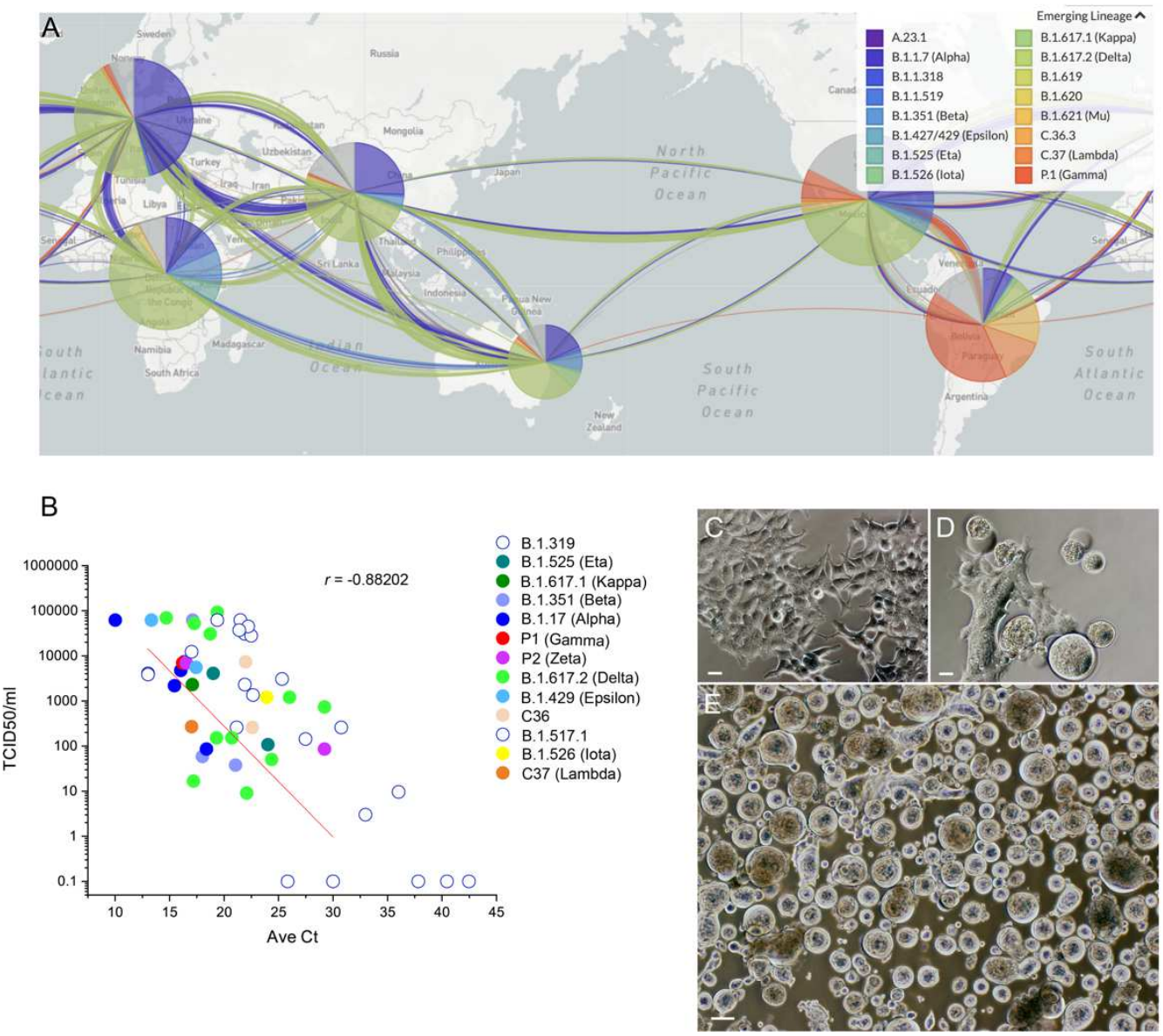

Figure 1: Sensitive isolation of all SARS-CoV-2 VOC and key VUI over the period of February to May $\underline{2021 .}$

(A) Summary of variants arriving into NSW quarantine over the period of February to May 2021. Transmission lines courtesy of Nextstrain and restricted to NSW and the above time period. (B) Rapid end-point titres versus average $\mathrm{Ct}$ values ( $\mathrm{Ct}$ values are averages of the $\mathrm{Ct}$ values across the viral genes detected: (i.e. Primarily E, RdRP and $\mathrm{N}$ ) recorded using the HAT-24 cell line for rapid and sensitive isolation of SARS-CoV-2. Variant B.1.517.1 (open blue circles) is used herein as a comparison, with samples derived from community spread in Australia from December 2020 to the end of January 2021. (C-E) Rapid appearance of viral CPE (see large spherical viral syncytia) following (D) overnight versus E. 48-hour culture with a swab positive for the VOC Beta (C is mock uninfected control). CPE appearance is representative of all VOC. Scale bars in C to E are at 10um. 
Figure 2

A

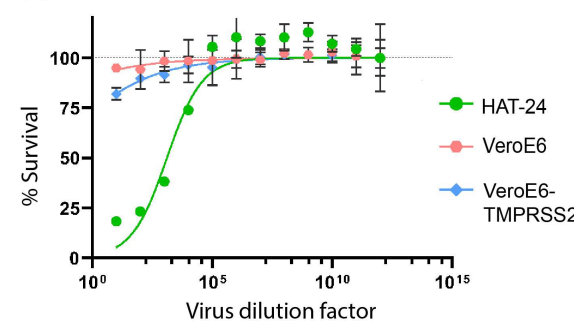

D

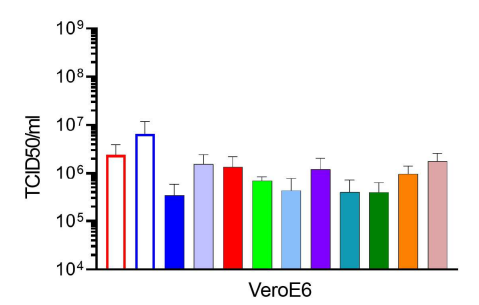

B

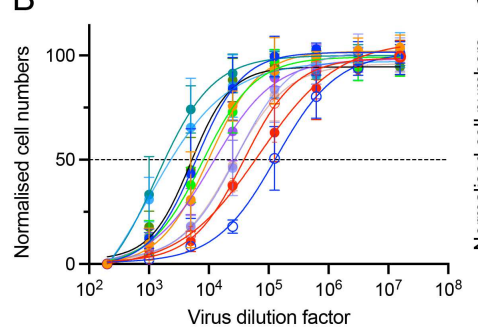

C

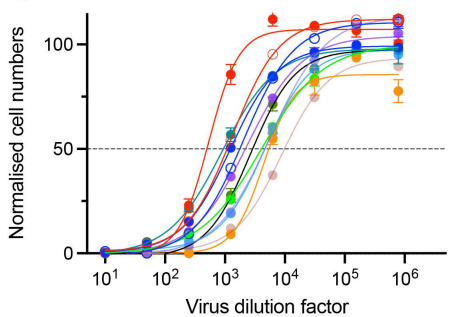

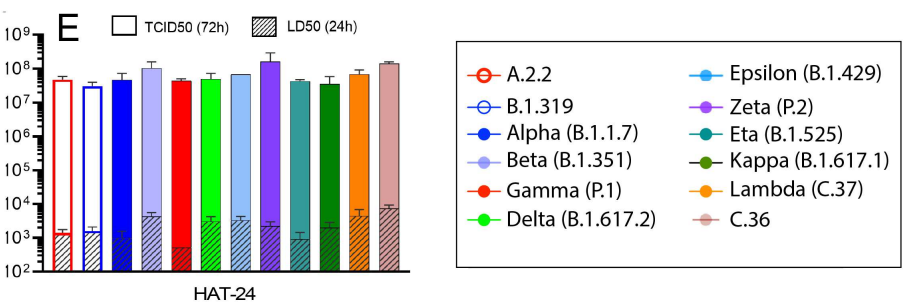

HAT-24

Figure 2: Titration of SARS-CoV-2 viral stocks in VeroE6 and HAT-24 cell lines.

Virus stocks were serially diluted in 5-fold steps and added to cells in octuplicate. Cell nuclei were enumerated with high content microscopy and cell numbers normalised to mock infected controls, where $100 \%$ represents cell numbers for mock-infected controls and $0 \%$ for the highest viral concentration (A) Dose dependent loss of nuclei in VeroE6-TMPRSS2, VeroE6 and HAT-24 cells at 20 hours post infection (hpi). (B-C) Titrations of a panel of SARS-CoV-2 isolates including the ancestral virus strain, variants of concern (VOC) and variants under investigation (VUI). Readout occurred 72 hpi for (B) VeroE6 and 20hpi for (C) HAT24 cells. (D-E) Visual scoring of cytopathic effect (CPE) at 72 hpi was done to calculate TCID50/ml values in (D) VeroE6 and (E) HAT24 cells (coloured bars). LD50 values at 20 hpi are also displayed (small bars with pattern). Data shown represents the mean and standard deviations from at least $\mathrm{n}=3$ experiments. 
Figure 3

A

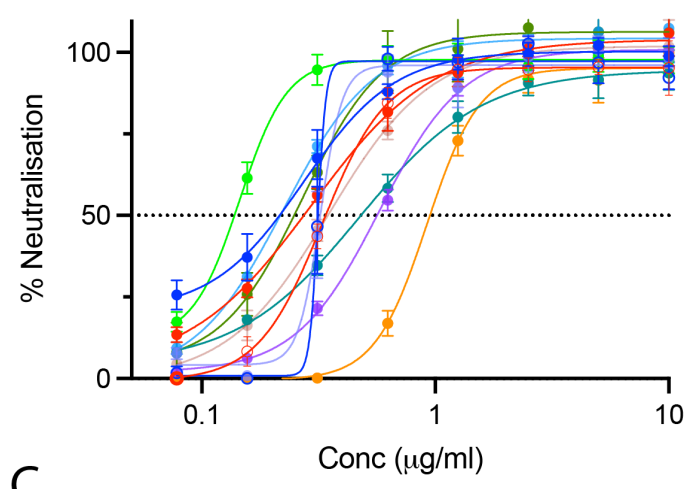

C

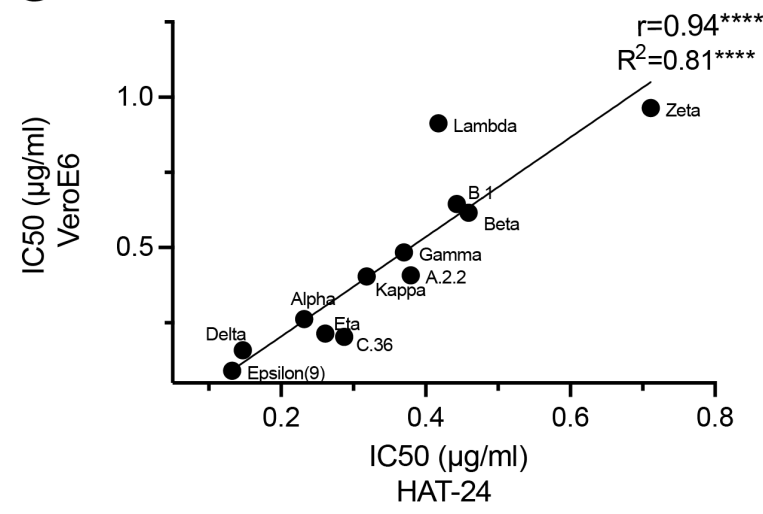

B

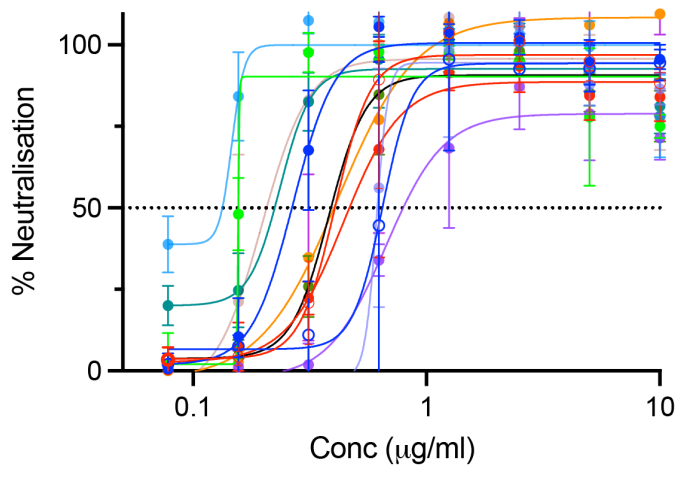

Figure 3: Cross-validation of the R-20 platform with monoclonal antibody 3467. Neutralisation assays were performed in high-throughput format with both VeroE6 cells and HAT-24 using live virus isolates from the variants of concern; Alpha (B.1.1.7), Beta (B.1.351), Gamma (P.1), and Delta (B.1.617.2), as well as the variants under investigation Epsilon (B.1.429), Zeta (P.2), Eta (B.1.525), Kappa (B.1.617.1), Lambda (C37), and C36. "Wildtype" virus from the same clade containing the dominant D614G mutation (B.1) and ancestral Wuhan-like virus with the original D614 background (A.2.2) were used as controls. (A-B) Neutralisation assay of SARSCoV-2 isolates with monoclonal Ab-3467 in (A) HAT-24, (B) VeroE6 and (C) Correlation of IC50 values in (AB) showing Spearman coefficient of correlation (r). ${ }^{*} p<0.05, * * p<0.01, * * * p<0.001,{ }^{* * * *} p<0.0001$. Shown are the mean and standard deviations from at least $\mathrm{n}=3$ experiments. 

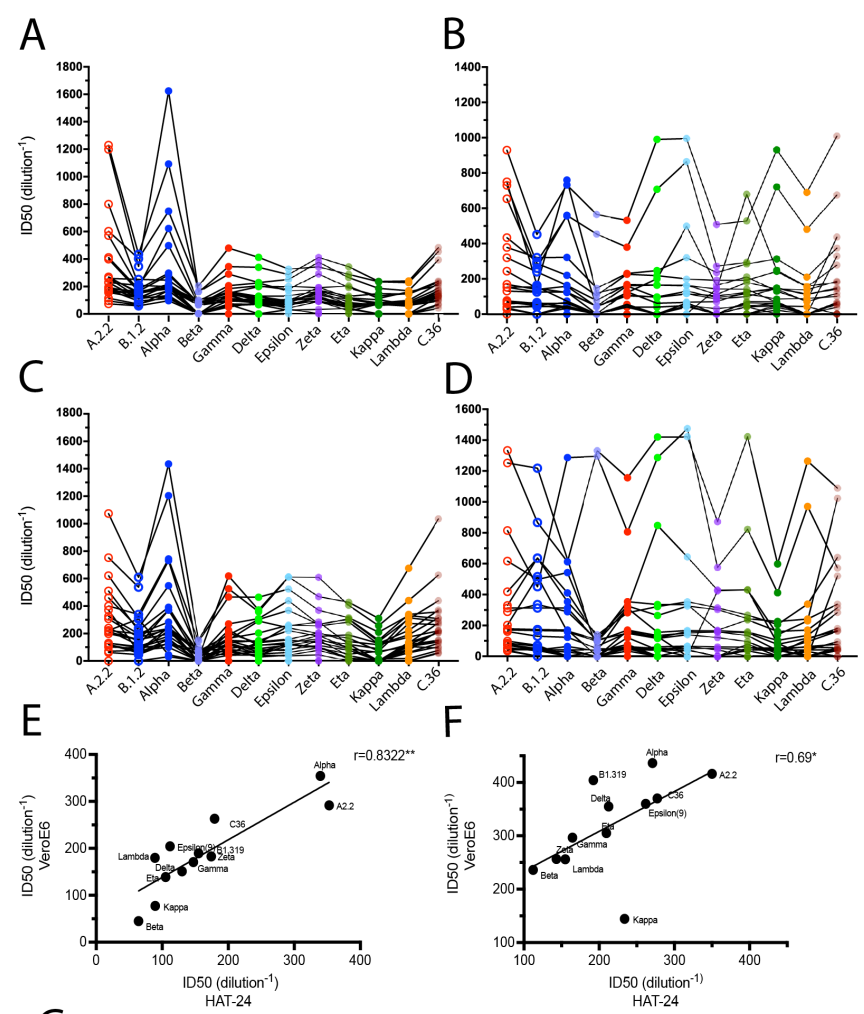

G
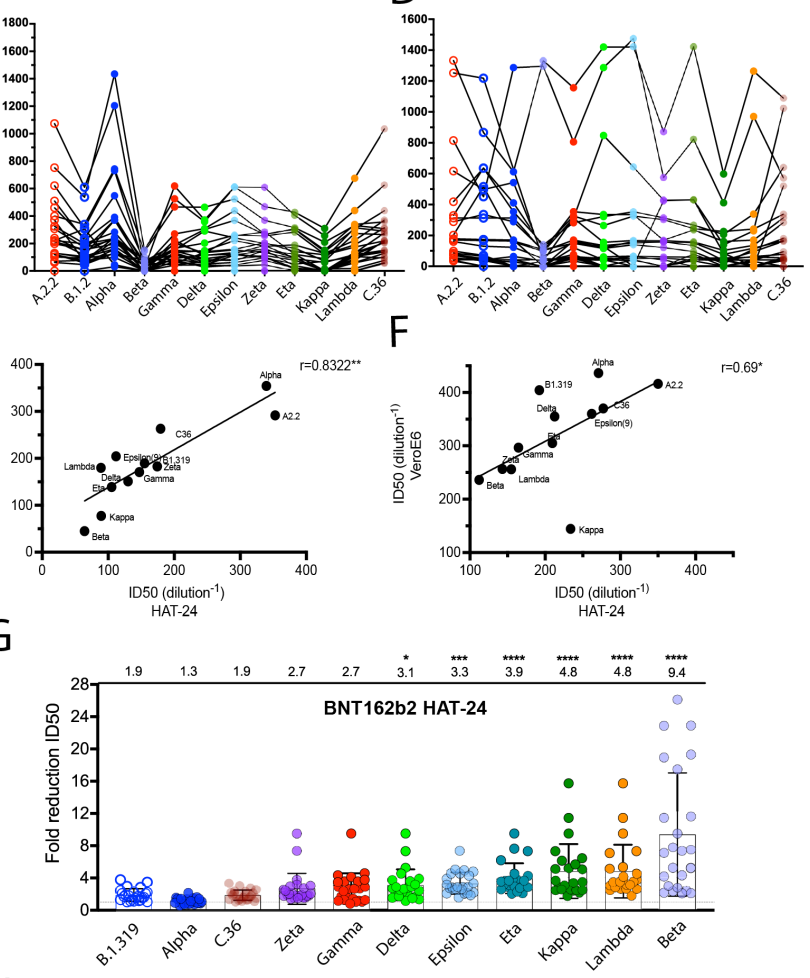

$\mathrm{H}$

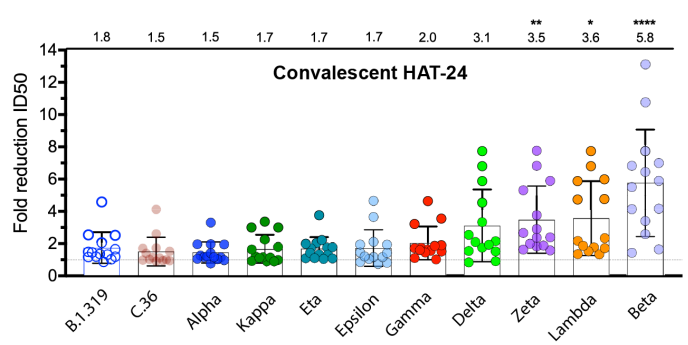

I

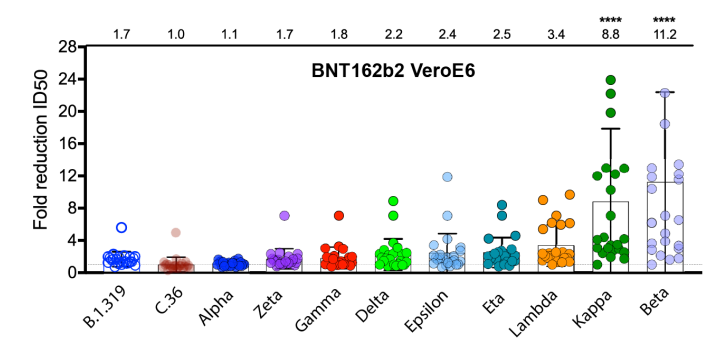

J

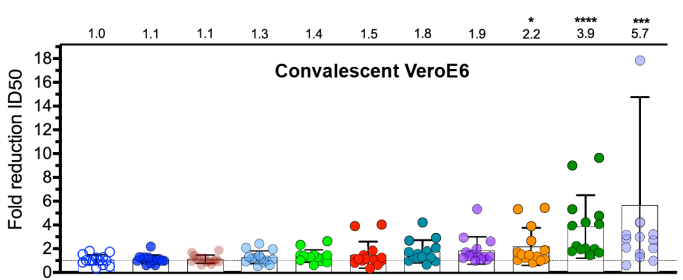

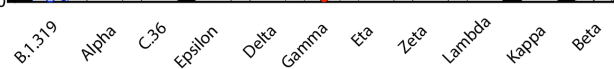


Figure 4: Contemporary live SARS-CoV-2 variants and evasion of the vaccine and convalescent response. (A-F) Neutralisation assays were performed in high-throughput format with both VeroE6 and HAT-24 cells using live virus isolates from the variants of concern; Alpha (B.1.1.7), Beta (B.1.351), Gamma (P.1), and Delta (B.1.617.2), as well as the variants under investigation Epsilon (B.1.429), Zeta (P.2), Eta (B.1.525), Kappa (B.1.617.1), Lambda (C37), and C36. "Wildtype" virus from the same clade containing the dominant D614G mutation (B.1) and ancestral Wuhan-like virus with the original D614 background (A.2.2) were used as controls. (A\&C). IC50 neutralisation titres presented for 12 live variants for peak BNT162b2-vaccinated samples and (B\&D) convalescent samples for HAT-24 and VeroE6, respectively. (E\&F) Correlation of IC50 values shown in $(\mathbf{A} \& \mathbf{C})$ and $(\mathbf{B} \& D)$ respectively showing Spearman coefficient of correlation $(\mathrm{r}){ }^{*} \mathrm{p}<0.05,{ }^{*} \mathrm{p}<0.01, * * * \mathrm{p}<0.001$, $* * * * \mathrm{p}<0.0001$. (G-J). Fold reduction in titres from data presented in (A-D). Columns indicate the mean fold reduction and standard deviation of all samples for each viral variant. $* \mathrm{p}<0.05, * * \mathrm{p}<0.01, * * * \mathrm{p}<0.001$, $* * * * \mathrm{p}<0.0001$ for Friedman's test with Dunn's multiple comparison test. 

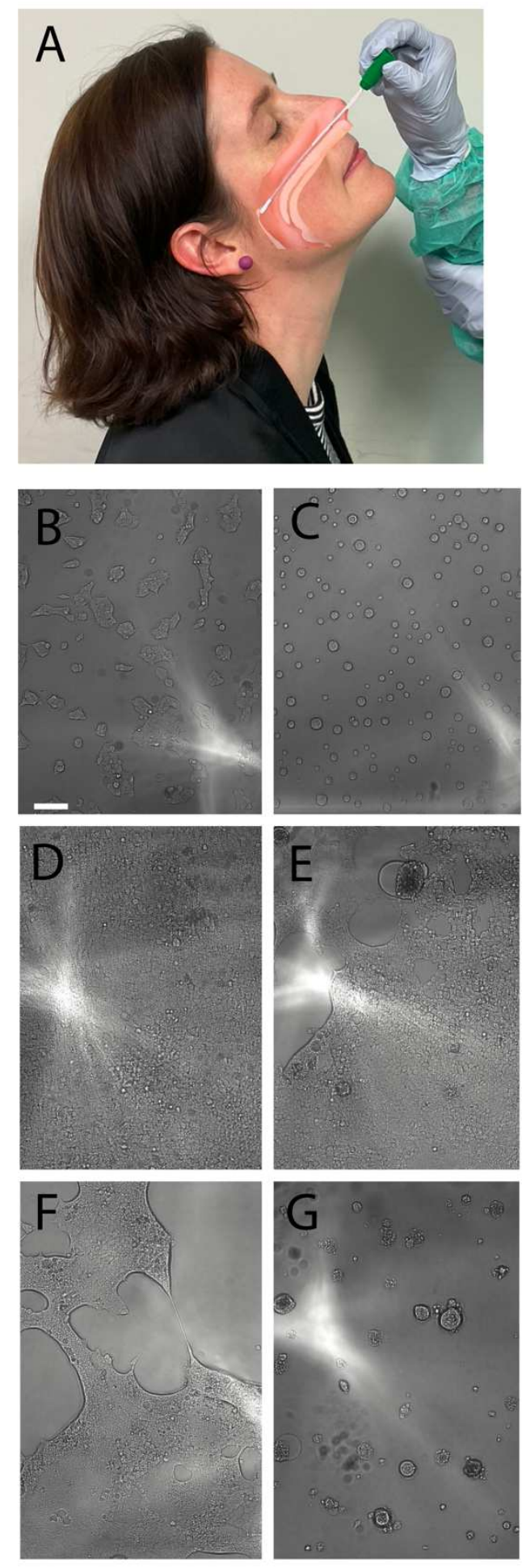

$\mathrm{H}$

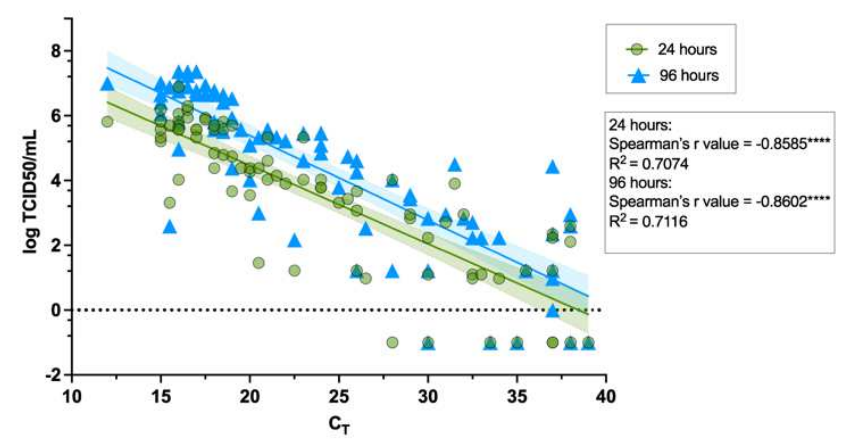

\footnotetext{
I
}

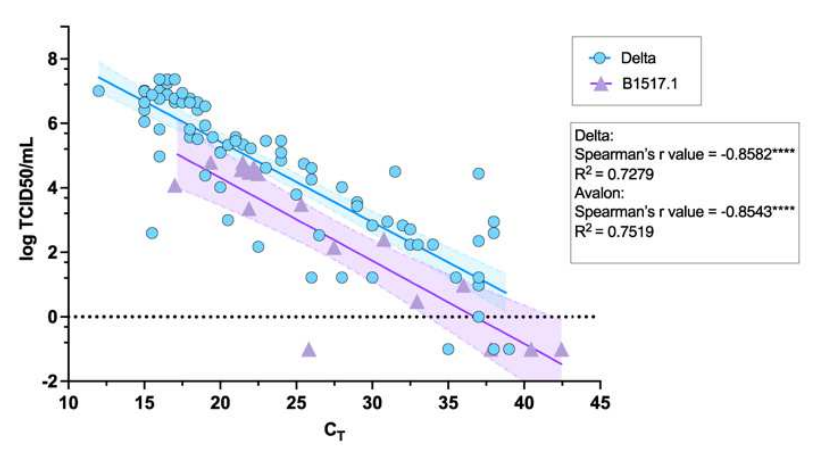

J
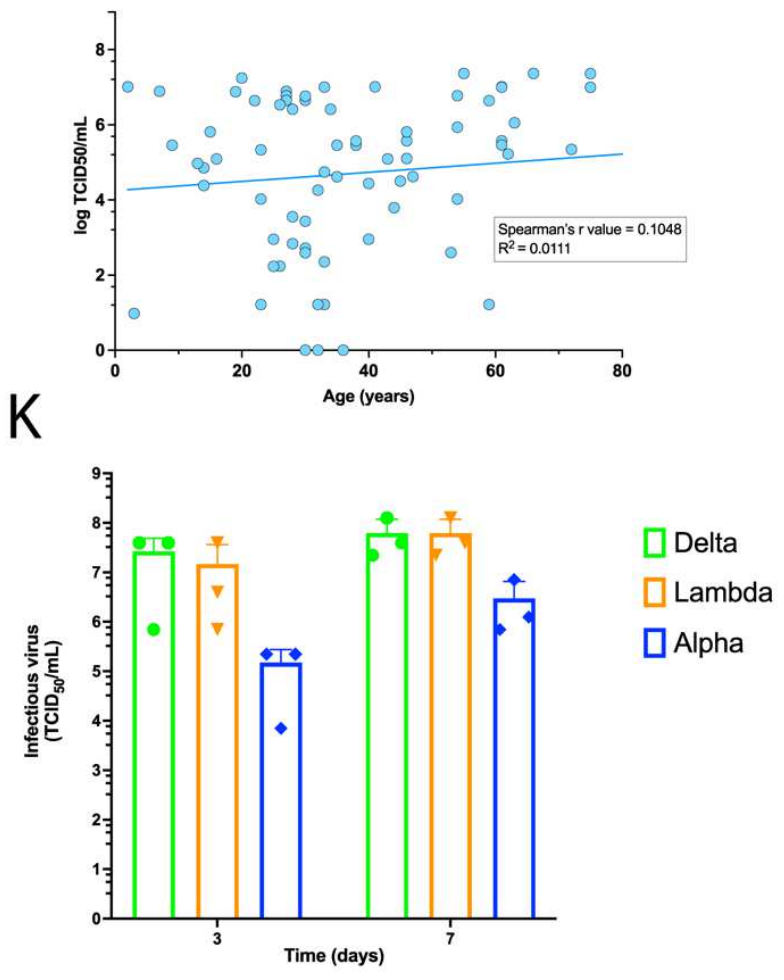

Figure 5: Resolution of variant fitness in vivo by combining rapid and sensitive end-point viral titres with diagnostic PCR values.

(A) Nasopharyngeal swabs were obtained over December 2020 to June 2021. In brief, samples were taken, placed into viral transport media and frozen at $-80^{\circ} \mathrm{C}$ in $100 \mu 1$ aliquots within 24 hours. (B-G) Samples were filter sterilised using 0.22 um centrifugal filters and the co-cultured with the HAT-24 line. Upper panels (20 hours of culture): Left, uninfected cells versus Right, infected cells with a swab $\mathrm{Ct}<20$. Note CPE is scored through the rapid appearance of large spherical syncytia. Middle panels ( 96 hours of culture), Left uninfected confluent well. Right panel, low level infection with CPE revealed as a combination of spherical syncytia and the formation of plaques. Lower panels (96 hours of culture), Left panel, low level infection with CPE revealed with extensive 
fusion across the viral cell sheet and the formation of plaques. Right panel, (96 hours post infection) extensive infection where CPE has resolved early as spherical syncytia and has over time become granular in appearance. (H) Rapid end-point titres versus average $\mathrm{Ct}$ values as described in Figure 1. Here the titres are scored within 20 hours versus 96 hours and correlated to the average $\mathrm{Ct}$ of all genes detected in diagnostic PCR values. All samples were collected as outlined in (A) and are from the Delta outbreak in Sydney June 2021. (I) Comparison of the early clade B.1.517.1 versus the VOC Delta. Swabs for B.1.517.1 were collected as outlined in (A) but during December 2020 to January 2021. (J) Correlation of Delta infectious titres versus age. (K) Titres derived from primary ALI cultures, 3 days and 7 days post infection using the R-20 platform.
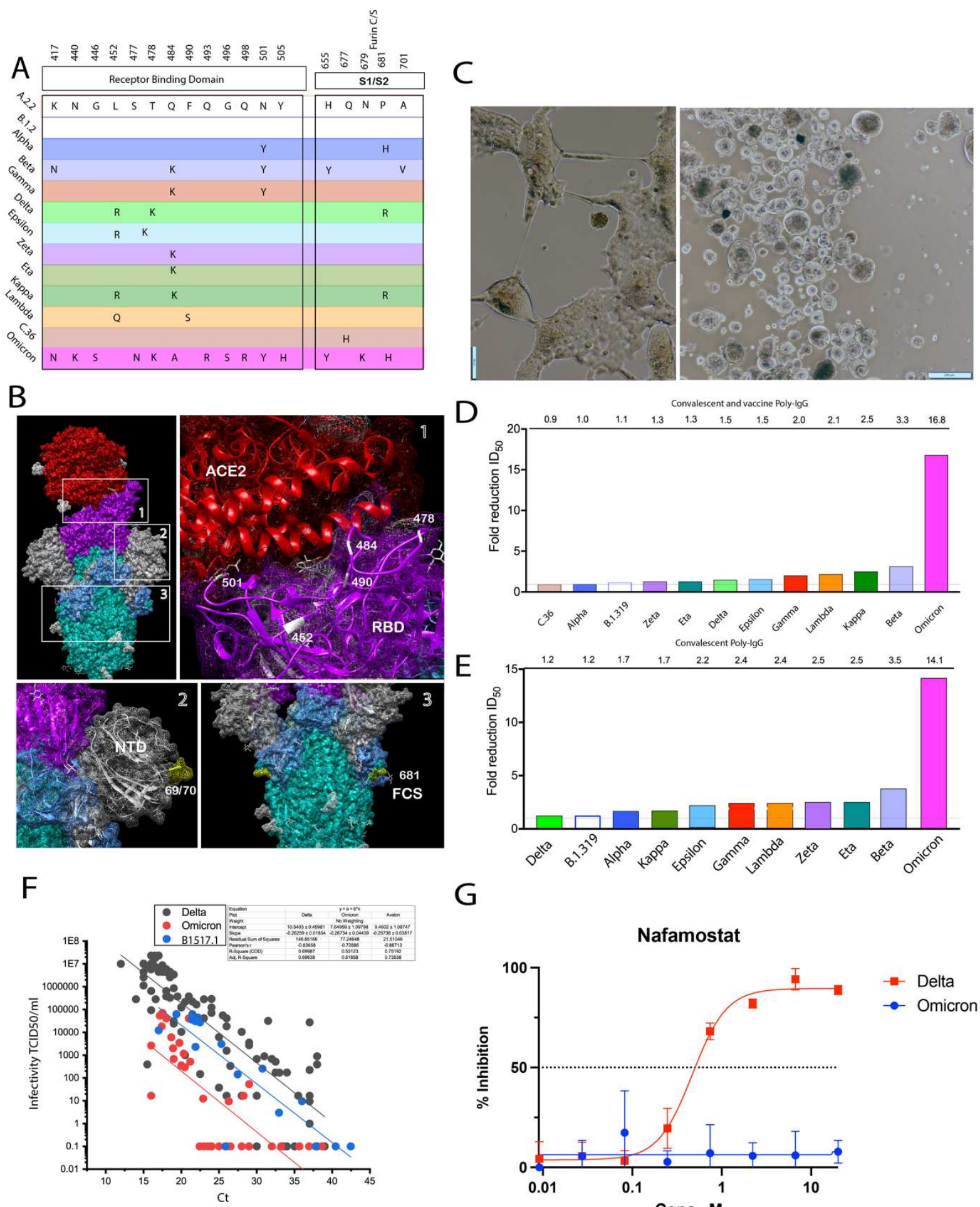

G

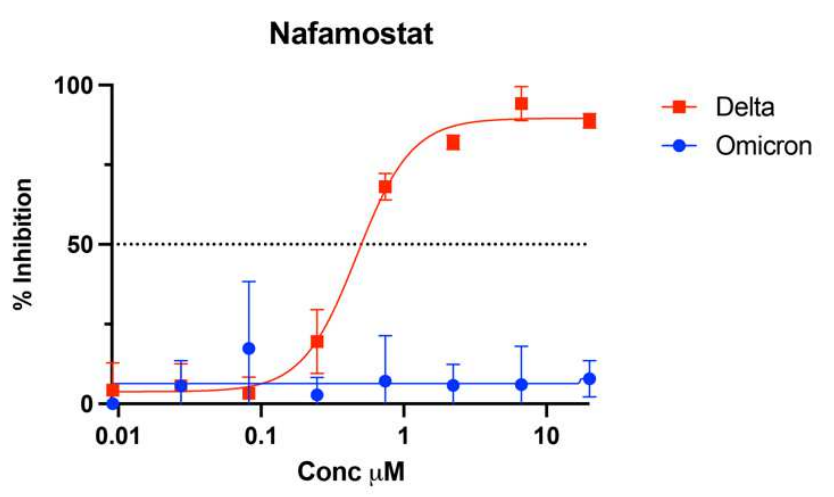

Figure 6: Rapid isolation and characterisation of the SARS-CoV-2 Omicron variant 
(A) Extensive changes in the RBD and furin cleavage site in Omicron versus all variants tested herein. (B) Key site changes within the RBD, N-terminal domain and furin cleavage site observed in variants tested herein. Note changes/deletions at positions del69/70 (N-terminal domain), 501, 484, 478 (RBD) and 681 (furin cleavage site) are shared sites between Omicron and other VOC. (C) Primary Omicron sample (Ct 22) was filtered sterilised using 0.22 um centrifugal filters and the co-cultured with the HAT-24 line. Left panel (48 hours of culture). Right panel 72 hours of culture. As with other SARS-CoV-2 variants extensive syncytia accumulates within the HAT24 line. (D) Fold-reduction of neutralisation potency across all variants tested herein using human polyclonal IgG derived from more than 20,000 US plasma donors. This represents the collective activity neutralising antibodies derived from both convalescent and vaccinated plasma donors. (E) Fold-reduction of neutralisation potency across all variants tested herein using human polyclonal IgG derived from COVID19 convalescent donors plasma donors 37. Fold reduction values are representative of fold reduction from $n=3$ independent experiments. (E) End-point titres of Omicron plotted against the diagnostic PCR Ct value as described in Figure legend 5. Delta and the early variant B.1.517.1 linear regressions are overlayed for a comparison. (F) Titration of the TMPRSS2 inhibitor Nafamostat using the HAT-24 cell line. Note the lack of inhibition in Omicron versus Delta at all concentrations tested.
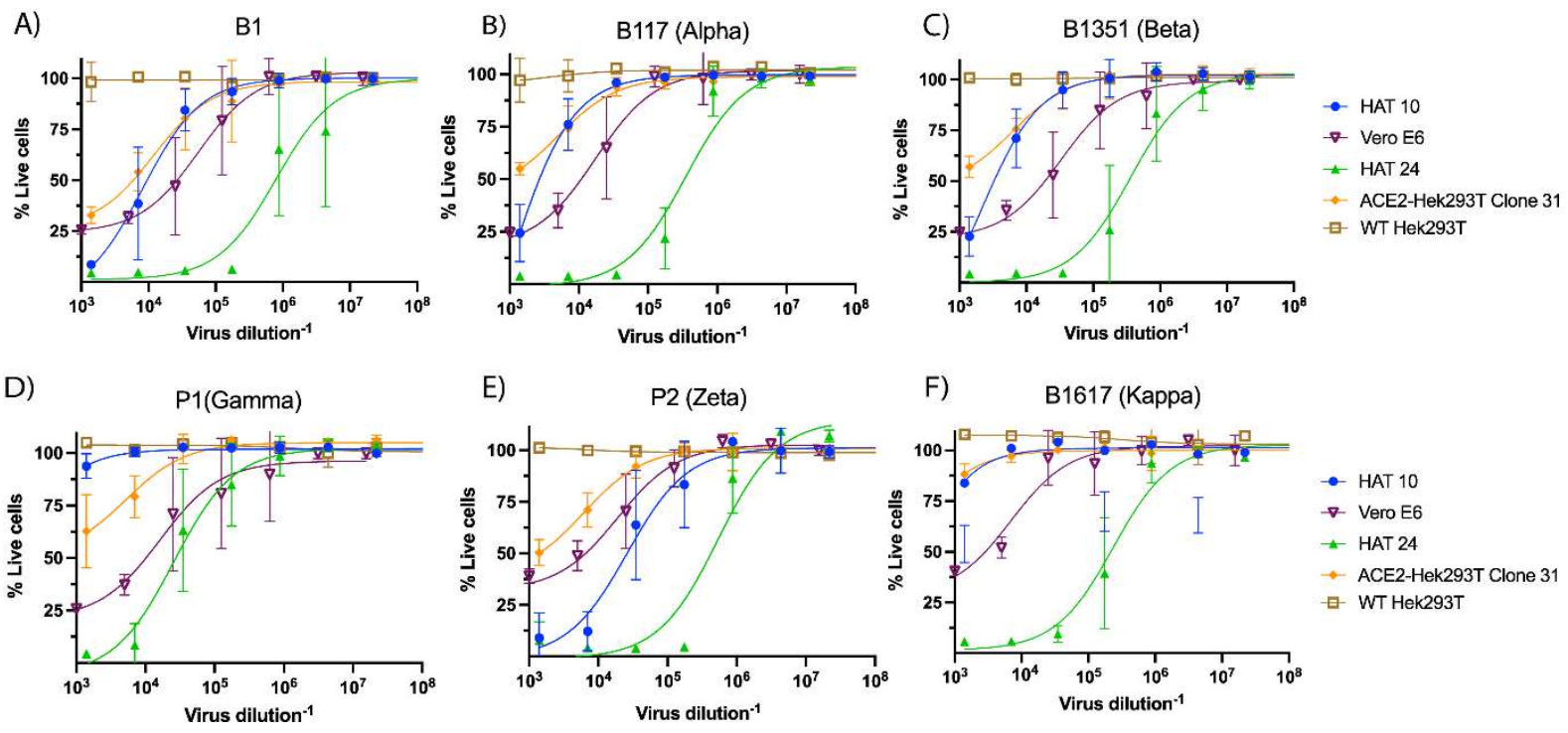

Supplementary Figure 1: Relative sensitivity of cellular clones to SARS-CoV-2 cytopathic effects.

Each cell indicated was infected with (A) early 2020 B1 clade B.1.319, (B) Alpha, (C) Beta, (D) Gamma, (E) Zeta and (F) Kappa to observe relative sensitivities to viral induced cytopathic effects (CPE). CPE was quantified 3 days post-infection by staining the remaining nuclei with the live nuclei stain Hoechst3342/NucBlue. All standard deviations and mean values are presented from 4 technical replicates per dilution. Clone 31 is a HEK293T clone engineered to express ACE2 only. HAT-10 is a HEK293T clone engineered to express ACE2 and TMPRSS2. Clone 10 was one of 23 clones that were observed to have an equivalent phenotype. HAT- 24 was the only clone out of 24 to be observed to be highly permissive to SARS-CoV-2 infection and sustained CPE within 8 hours post-infection. Parental HEK293T cells and VeroE6 cells are presented are a comparison. Data is representative of $>\mathrm{n}=10$ independent experiments.

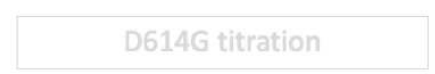

A)

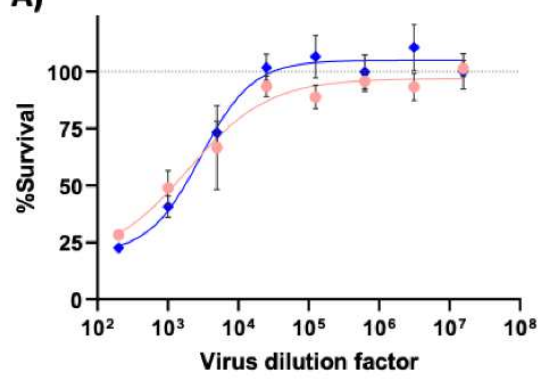

B)

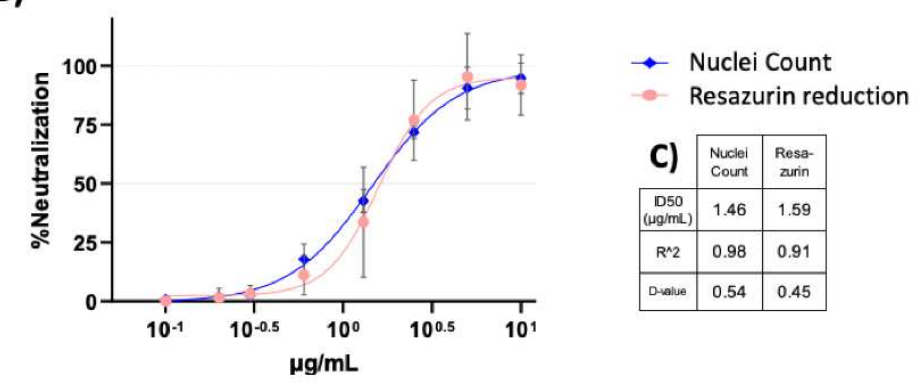


Supplementary Figure 2: Alternate low cost readout for rapid SARS-CoV-2 live virus neutralisation assay. (A) Viral titration and (B) neutralisation assays using the human monoclonal antibody 3467 (Ab-3467) were validated for an alternative readout method using metabolic reduction of resazurin to simplify the high content read out to a standard optical plate reader. HAT-24 cells were either pre-stained before seeding with Hoechst 3342 nuclear dye for end-point readout via high-content microscopy and automated whole-well nuclei counts (blue curve) or seeded unstained for end-point readout via 3 hour resazurin reduction and whole-well fluorescence measurement (pink curve). Cells were seeded in 96-well plates at $6.4 \times 10^{\wedge} 4$ cells/well and received an equal volume of B.1.319 virus in increasing concentrations (A), or a fixed amount of virus previously incubated for 1 hour with increasing concentrations of the monoclonal antibody Ab-3467. (B) Plates were incubated for 20 hours at $37^{\circ} \mathrm{C}$ until end-point readout, and percentage of survival was calculated relative to average signal from uninfected controls, with percentage of neutralisation calculated as described in the methods section. 0 C) $50 \%$ inhibitory dose (ID50) for Ab-3467 and the dose-dependent sigmoidal fit (R-squared value) for the fitted sigmoidal dose-response curves in (B). 
A)

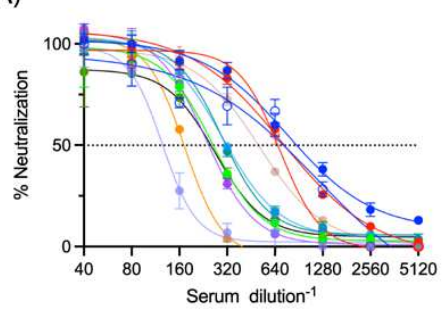

D)

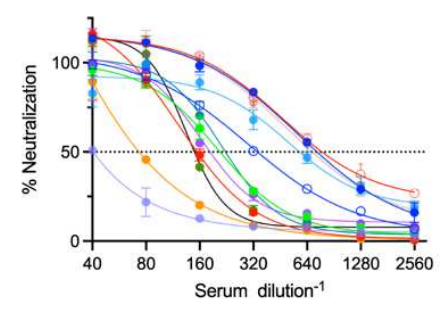

G)

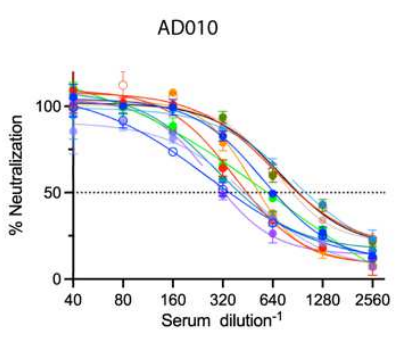

J)

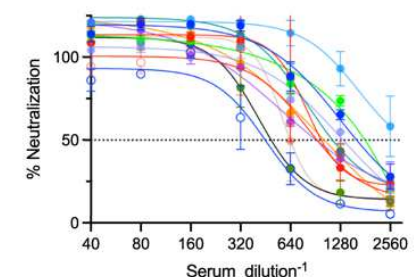

$$
\begin{array}{ll}
\rightarrow \text { B1 } & \rightarrow \text { B1429 (Epsilon) } \\
\rightarrow \text { B1.2 } & \rightarrow \text { P2 (Zeta) } \\
\rightarrow \text { B117 (Alpha) } & \rightarrow \text { B1525 (Eta) } \\
\rightarrow \text { B1351 (Beta) } & \rightarrow \text { B1617 (Kappa) } \\
\rightarrow \text { P1(Gamma) } & \rightarrow \text { C37 (Lambda) } \\
\rightarrow \text { B1617.2 (Delta) } & \rightarrow \text { C36 }
\end{array}
$$

Supplementary Figure 3: Representative breadth of donor specific responses for 12 primary variants.

(A) Representative BNT162b2 donor in (A) HAT-24 R-20 format versus (B) VeroE6 cells. (C) Correlation between HAT-24 and VeroE6 IC50 values from (A-B). (D-F) Representative convalescent donor with an eroding response to contemporary SARS-CoV-2. (D) R-20 format versus (E) VeroE6 (F) with respective IC50 value correlations for (D-E). (G, H, J, K) Neutralisation responses in two convalescent donors with good breadth across all variants in $(\mathbf{G} \& \mathbf{H})$ HAT-24 and $(\mathbf{J} \& \mathbf{K})$ VeroE6. (I \& L) Donor with initial good response to early clades but lack of end point titres to Gamma, Beta and variants with the spike L454R or L452Q (Delta, Kappa, Lambda and Epsilon) in HAT-24 and Vero-E6, respectively. Shown are the mean and standard deviations from 3 independent experiments.

Supplementary Table S1. IC50 values of Vaccine sera against SARS-COV2 isolates in HAT-
B)

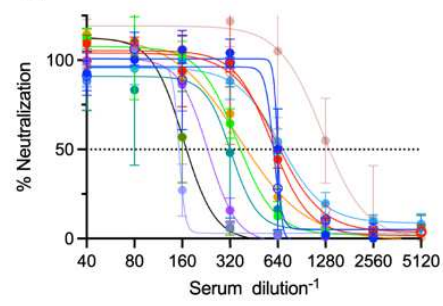

E)

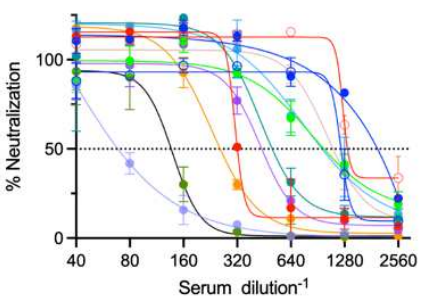

H)

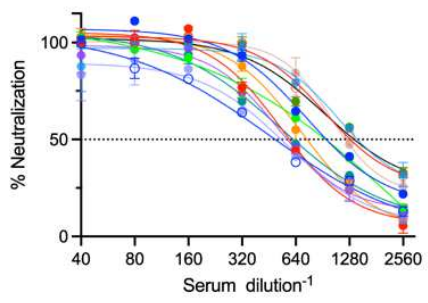

$\mathrm{K})$

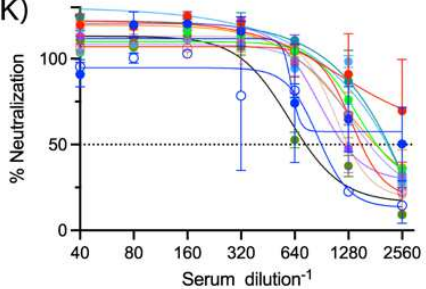

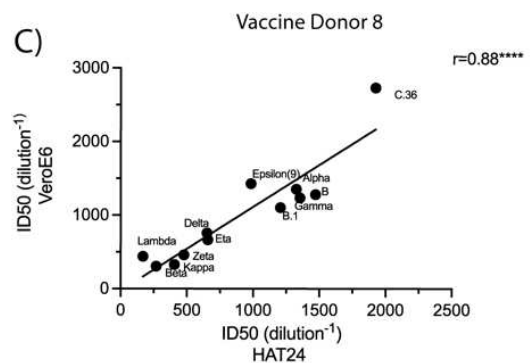

F)

l)

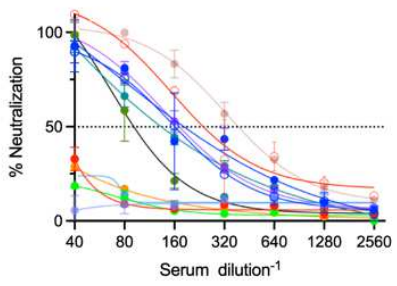

L)

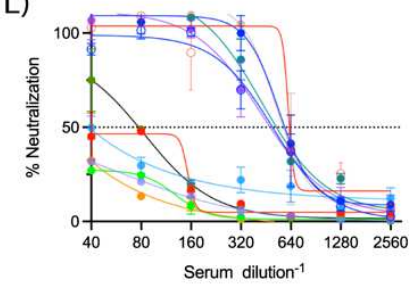
24. 
Supplementary Table S2. IC50 values of convalescent sera against SARS-COV2 isolates in HAT-24.

Supplementary Table S3. IC50 values of Vaccine sera against SARS-COV2 isolates in VeroE6.

Supplementary Table S4: IC50 values of convalescent sera against SARS-COV2 isolates in VeroE6.

Supplementary Table S5. Summary of NT50 values of Vaccine sera against SARS-COV2 isolates in HAT-24 and VeroE6.

Supplementary Table S6. Summary NT50 values of Convalescent sera against SARS-COV2 isolates in HAT-24 and VeroE6.

Supplementary Table S7. Genomic viral sequences for SARS-CoV-2 variants used in this study.

Supplementary Movie S1. Real-time formation of cytopathic effects within the HAT-24 cell line. Images are acquired using the Deltavision Elite microscope fitted with a 0.6 NA 20x Olympus objective. Images are acquired using a PhotometrixCoolSnap Camera with white light at $37^{\circ} \mathrm{C}$ supplemented with humidified $5 \% \mathrm{CO}_{2}$ at a frame rate of 1 frame per 3 minutes (total time 20 hours). Scale bar in video is in the lower left corner at $25 \mathrm{um}$. Note that cytopathic effects appear at the 8 hour time point. 


\section{References Cited}

1 Davies, N. G. et al. Estimated transmissibility and impact of SARS-CoV-2 lineage B.1.1.7 in England. Science (New York, N.Y.) 372, doi:10.1126/science.abg3055 (2021).

2 Faria, N. R. et al. Genomics and epidemiology of the P.1 SARS-CoV-2 lineage in Manaus, Brazil. Science (New York, N.Y.) 372, 815-821, doi:10.1126/science.abh2644 (2021).

3 Tegally, H. et al. Detection of a SARS-CoV-2 variant of concern in South Africa. Nature 592, 438-443, doi:10.1038/s41586-021-03402-9 (2021).

4 Mlcochova, P. et al. SARS-CoV-2 B.1.617.2 Delta variant replication and immune evasion. Nature, doi:10.1038/s41586-021-03944-y (2021).

5 Shinde, V. et al. Efficacy of NVX-CoV2373 Covid-19 Vaccine against the B.1.351 Variant. N Engl J Med 384, 1899-1909, doi:10.1056/NEJMoa2103055 (2021).

6 Madhi, S. A. et al. Efficacy of the ChAdOx1 nCoV-19 Covid-19 Vaccine against the B.1.351 Variant. N Engl J Med 384, 1885-1898, doi:10.1056/NEJMoa2102214 (2021).

7 Liu, Y. et al. Delta spike P681R mutation enhances SARS-CoV-2 fitness over Alpha variant. bioRxiv, doi:10.1101/2021.08.12.456173 (2021).

8 Reardon, S. How the Delta variant achieves its ultrafast spread. Nature, doi:10.1038/d41586-021-01986-w (2021).

9 Madewell, Z. J., Yang, Y., Longini, I. M., Jr., Halloran, M. E. \& Dean, N. E. Factors Associated With Household Transmission of SARS-CoV-2: An Updated Systematic Review and Meta-analysis. JAMA Netw Open 4, e2122240, doi:10.1001/jamanetworkopen.2021.22240 (2021).

10 Bull, R. A. et al. Analytical validity of nanopore sequencing for rapid SARS-CoV-2 genome analysis. Nat Commun 11, 6272, doi:10.1038/s41467-020-20075-6 (2020).

11 Hoffmann, M. et al. SARS-CoV-2 Cell Entry Depends on ACE2 and TMPRSS2 and Is Blocked by a Clinically Proven Protease Inhibitor. Cell 181, 271-280 e278, doi:10.1016/j.cell.2020.02.052 (2020).

12 Ferreira, C. B. et al. Lentiviral Vector Production Titer Is Not Limited in HEK293T by Induced Intracellular Innate Immunity. Mol Ther Methods Clin Dev 17, 209-219, doi:10.1016/j.omtm.2019.11.021 (2020).

13 Ronco, L. V., Karpova, A. Y., Vidal, M. \& Howley, P. M. Human papillomavirus 16 E6 oncoprotein binds to interferon regulatory factor-3 and inhibits its transcriptional activity. Genes Dev 12, 2061-2072, doi:10.1101/gad.12.13.2061 (1998).

14 Fonseca, G. J. et al. Adenovirus evasion of interferon-mediated innate immunity by direct antagonism of a cellular histone posttranslational modification. Cell host \& microbe 11, 597-606, doi:10.1016/j.chom.2012.05.005 (2012).

15 Bhattacharya, S. et al. Cooperation of Stat2 and p300/CBP in signalling induced by interferon-alpha. Nature 383, 344-347, doi:10.1038/383344a0 (1996). 
16 Juang, Y. T. et al. Primary activation of interferon A and interferon B gene transcription by interferon regulatory factor 3. Proceedings of the National Academy of Sciences of the United States of America 95, 9837-9842, doi:10.1073/pnas.95.17.9837 (1998).

17 Ramakrishnan, M. A. Determination of 50\% endpoint titer using a simple formula. World J Virol 5, 85-86, doi:10.5501/wjv.v5.i2.85 (2016).

18 Matsuyama, S. et al. Enhanced isolation of SARS-CoV-2 by TMPRSS2-expressing cells. Proceedings of the National Academy of Sciences of the United States of America 117, 7001-7003, doi:10.1073/pnas.2002589117 (2020).

19 Armitage, P. \& Allen, I. Methods of estimating the LD 50 in quantal response data. $J$ Hyg (Lond) 48, 298-322, doi:10.1017/s0022172400015084 (1950).

20 Tea, F. et al. SARS-CoV-2 neutralizing antibodies: Longevity, breadth, and evasion by emerging viral variants. PLoS Med 18, e1003656, doi:10.1371/journal.pmed.1003656 (2021).

21 Burnett, D. L. et al. Immunizations with diverse sarbecovirus receptor-binding domains elicit SARS-CoV-2 neutralizing antibodies against a conserved site of vulnerability. Immunity 54, 2908-2921 e2906, doi:10.1016/j.immuni.2021.10.019 (2021).

22 Liu, J. et al. BNT162b2-elicited neutralization of B.1.617 and other SARS-CoV-2 variants. Nature 596, 273-275, doi:10.1038/s41586-021-03693-y (2021).

23 Zhou, D. et al. Evidence of escape of SARS-CoV-2 variant B.1.351 from natural and vaccine-induced sera. Cell 184, 2348-2361 e2346, doi:10.1016/j.cell.2021.02.037 (2021).

24 Edara, V. V. et al. Infection- and vaccine-induced antibody binding and neutralization of the B.1.351 SARS-CoV-2 variant. Cell host \& microbe 29, 516-521 e513, doi:10.1016/j.chom.2021.03.009 (2021).

25 Shen, X. et al. Neutralization of SARS-CoV-2 Variants B.1.429 and B.1.351. N Engl J Med 384, 2352-2354, doi:10.1056/NEJMc2103740 (2021).

26 Planas, D. et al. Sensitivity of infectious SARS-CoV-2 B.1.1.7 and B.1.351 variants to neutralizing antibodies. Nat Med 27, 917-924, doi:10.1038/s41591-021-01318-5 (2021).

27 Loo, S. L. et al. Human coronaviruses 229E and OC43 replicate and induce distinct antiviral responses in differentiated primary human bronchial epithelial cells. $A m \mathrm{~J}$ Physiol Lung Cell Mol Physiol 319, L926-L931, doi:10.1152/ajplung.00374.2020 (2020).

28 Campbell, F. et al. Increased transmissibility and global spread of SARS-CoV-2 variants of concern as at June 2021. Euro Surveill 26, doi:10.2807/15607917.ES.2021.26.24.2100509 (2021).

29 Planas, D. et al. Reduced sensitivity of SARS-CoV-2 variant Delta to antibody neutralization. Nature 596, 276-280, doi:10.1038/s41586-021-03777-9 (2021).

30 Lopez Bernal, J. et al. Effectiveness of Covid-19 Vaccines against the B.1.617.2 (Delta) Variant. N Engl J Med 385, 585-594, doi:10.1056/NEJMoa2108891 (2021).

31 Johnson, B. A. et al. Loss of furin cleavage site attenuates SARS-CoV-2 pathogenesis. Nature 591, 293-299, doi:10.1038/s41586-021-03237-4 (2021).

32 Lukassen, S. et al. SARS-CoV-2 receptor ACE2 and TMPRSS2 are primarily expressed in bronchial transient secretory cells. The EMBO journal 39, e105114, doi:10.15252/embj.20105114 (2020).

33 Chau, N. V. V. et al. An observational study of breakthrough SARS-CoV-2 Delta variant infections among vaccinated healthcare workers in Vietnam. EClinicalMedicine 41, 101143, doi:10.1016/j.eclinm.2021.101143 (2021). 
34 Pouwels, K. B. et al. Effect of Delta variant on viral burden and vaccine effectiveness against new SARS-CoV-2 infections in the UK. Nat Med, doi:10.1038/s41591-02101548-7 (2021).

35 Bergwerk, M. et al. Covid-19 Breakthrough Infections in Vaccinated Health Care Workers. N Engl J Med 385, 1474-1484, doi:10.1056/NEJMoa2109072 (2021).

$36 \mathrm{Lu}, \mathrm{Z}$. et al. Evolution of HIV-1 coreceptor usage through interactions with distinct CCR5 and CXCR4 domains. Proceedings of the National Academy of Sciences of the United States of America 94, 6426-6431, doi:10.1073/pnas.94.12.6426 (1997).

37 Kober, C. et al. IgG3 and IgM Identified as Key to SARS-CoV-2 Neutralization in Convalescent Plasma Pools. PLoS One 17, e0262162, doi:10.1371/journal.pone.0262162 (2022).

38 Stucki, M. et al. Investigations of prion and virus safety of a new liquid IVIG product. Biologicals 36, 239-247, doi:10.1016/j.biologicals.2008.01.004 (2008).

39 Karbiener, M. et al. Plasma from post-COVID-19 and COVID-19-Vaccinated Donors Results in Highly Potent SARS-CoV-2 Neutralization by Intravenous Immunoglobulins. J Infect Dis, doi:10.1093/infdis/jiab482 (2021).

40 Kristiansen, P. A. et al. WHO International Standard for anti-SARS-CoV-2 immunoglobulin. Lancet 397, 1347-1348, doi:10.1016/S0140-6736(21)00527-4 (2021).

41 Follenzi, A. et al. Targeting lentiviral vector expression to hepatocytes limits transgenespecific immune response and establishes long-term expression of human antihemophilic factor IX in mice. Blood 103, 3700-3709, doi:10.1182/blood-2003-093217 (2004).

42 Workshop summary and guidelines: investigative use of bronchoscopy, lavage, and bronchial biopsies in asthma and other airway diseases. J Allergy Clin Immunol 88, 808-814, doi:0091-6749(91)90189-U [pii] (1991). 


\section{$\underline{\text { Resource Availability }}$}

\section{Lead contact}

Further information and requests for resources and reagents should be directed to the lead contact, Stuart Turville (sturville@kirby.unsw.edu.au).

\section{Materials availability}

This study generated a new reagent described as HEK293T-ACE2-TMPRSS2-Clone\#24 (HAT-24) cell line. HAT-24 cells will be made available through the Cell-Bank Australia or directly through the corresponding author through a material transfer agreement.

\section{Data and code availability}

Whole-genome viral sequences for the SARS-CoV-2 variants used in this study have been deposited to GISAID and are publicly available as of the date of publication. Accession numbers are listed in Table 1. IC50 values for the BNT162b2-vaccinated cohort are listed in Supplementary Table S7, and end-point titre values for the convalescent ADAPT cohort are listed in Supplementary Tables S1-6. Any additional data in this paper can be shared by the lead contact upon request. This paper does not report original code.

\section{Experimental model and subject details}

\section{Cell culture}

HEK293T cells (Thermofisher Scientific, \#R70007), HEK293T derivatives, and VeroE6TMPRSS2 (CellBank Australia, JCRB1819) were cultured in Dulbecco's Modified Eagle Medium (Gibco, 11995073) with 10\% FBS (Sigma, \#F423-500). VeroE6 cells (ATCC® CRL$1586^{\mathrm{TM}}$ ) were cultured in Minimum Essential Medium (Sigma, M4655) with 10\% FBS, and 1\% Penicillin-Streptomycin (Gibco, 15140122). Primary bronchial epithelial cell cultures (pBECs) were provided by P. A. B. Wark (University of Newcastle), and originally obtained from one healthy non-smoking donor (73-year-old female) during bronchoscopy, with written informed consent. pBECs were grown and differentiated until confluent in complete Bronchial Epithelial Cell Growth Basal Medium (Lonza, CC-3171) prior to use for air-liquid interface experiments. All cells were cultured and incubated at $37^{\circ} \mathrm{C}, 5 \% \mathrm{CO}_{2}$ and $>90 \%$ relative humidity unless otherwise indicated.

\section{Participants and patient samples}


Four cohorts of human study participants were considered in this study for studying neutralisation of viral variants. The Adapting to Pandemic Threats (ADAPT) cohort is composed of RT-PCR-confirmed convalescent individuals recruited during 2020 in Australia [PMID: 34228725]. Of the $>200$ ADAPT participants, a panel of 25 representative donors was chosen based on serum neutralisation titres against 'wildtype' B.1-clade virus (4 donors each for end-point titres of: $\leq 40,40,80,160,320$ and $\geq 640$ ). A second cohort was composed of 24 healthy adult vaccine-recipients, who received the BNT162b2 vaccine in 2021. Serum samples were collected three weeks post second-dose vaccination. Nasopharyngeal swabs were collected either from returning travellers entering Australian quarantine or during community spread following single viral seeding events leading to expansion of the B.1.517.1 variant in December 2020 in Sydney and also in July 2021, also in Sydney, during the first Delta outbreak (B.1.617.2) in Australia. All primary swabs were deidentified following diagnosis and frozen within 24 hours at $-80^{\circ} \mathrm{C}$. The approval of use remnant diagnostic swabs for research purposes was obtained through the New South Wales Chief Health Officer following independent scientific review.

\section{Polyclonal Immunoglobulin preparations and anti-SARS-CoV-2 hyperimmune globulin}

A CoVIg-19 Plasma Alliance (Poly IgG Convalescent 869) was formed in 2020 between major plasma pharmaceuticals including CSL, Takeda, Octapharma and Sanquin with an aim to develop a COVID-19 immunoglobulin therapy. As part of that initiative, CSL Behring manufactured anti-SARS-CoV-2 hyperimmune globulin (CoVIg). Approximately 5000 convalescent donor plasma units were collected between September and October 2020, exclusively from SARS-CoV-2 convalescent donors after COVID-19 confirmation ${ }^{37}$, the immunoglobulin purified using the licensed and fully validated immunoglobulin manufacturing process used for Privigen ${ }^{38}$, notionally similar to others ${ }^{39}$. IVIG lots (Poly IgG 1033) manufactured using the Privigen process described by Stucki et al ${ }^{38}$ included US plasma collected by plasmapheresis from a mixture of vaccinated with SARS-CoV-2 mRNA vaccines, convalescent and non-convalescent donors (source plasma, $\mathrm{n}$ between 9495-23,667 per batch) majority of donations collected between April and June 2021. The WHO international reference standard for SARS-CoV-2 neutralisation (NIBSC 20/136) was obtained from ${ }^{40}$.

\section{Method Details}




\section{Generation of HEK293T-ACE2-TMPRSS2 cells (clone HAT-24)}

HEK293T cells stably expressing human ACE2 and TMPRSS2 were generated by lentiviral transductions as previously described ${ }^{20}$. Briefly, the ORFs for hACE2 (Addgene, \#1786) and hTMPRSS2a (IDT, synthetic gene fragment) were cloned into lentiviral expression vectors pRRLsinPPT.CMV.GFP.WPRE 41 and pLVX-IRES-ZsGreen (Clontech, 632187) respectively. For ACE2 cloning, Age1/Bsrg1 cut sites were used to replace GFP with ACE2 while hTMPRSS2a was cloned into pLVX-IRES-ZsGreen using EcoR1/XhoI restriction sites. Lentiviral particles expressing the above genes were produced by co-transfecting expression plasmids individually with a 2nd generation lentiviral packaging construct psPAX2 (courtesy of Dr Didier Trono through NIH AIDS repository) and VSVG plasmid pMD2.G (Addgene\#2259) in HEK293T producer cells using polyethyleneimine as previously described (PMID: 22685410). Virus supernatant was harvested 72 hours post transfection, pre-cleared of cellular debris and centrifuged at $28,000 x g$ for 90 minutes at $4{ }^{\circ} \mathrm{C}$ to generate concentrated virus stocks. Two successive rounds of lentiviral transductions were then performed on HEK293T cells to generate ACE-TMPRSS2aHEK293T cells. Clonal selection led to the identification of a highly permissive clone (HAT-24), which was then used in subsequent experiments ${ }^{20}$.

\section{Viral isolation from primary specimens}

Respiratory specimens were collected and stored at $4{ }^{\circ} \mathrm{C}$ for same-day diagnostic RT-qPCR (Allplex SARS-CoV-2 Assay, Seegene, South Korea). Specimens positive for SARS-CoV-2 were then frozen at $-80^{\circ} \mathrm{C}$ within 24 hours of collection, and later transported to a certified BSL-3 facility, for primary isolate propagation. Thawed viral eluate was sterile-filtered through $0.22 \mu \mathrm{m}$ column-filters (Merck \#UFC30GVOS) at 10,000 xg for 5 minutes and then serially diluted (3-fold series) in quadruplicate. Viral dilutions were added to HAT-24 cells seeded in 96-well plates at $10^{4}$ cells/well seeded in a 96-well plate (final volume $100 \mu \mathrm{L}$ ). Plates were incubated at $37^{\circ} \mathrm{C}$ and monitored by brightfield microscopy every 24 hours using high content microscopy. Once extensive cytopathic effects became evident in at least 2 dilutions (see Figure 1E), the cells and supernatant from those cultures were collected and cleared from debris by centrifugation at $2000 \mathrm{xg}$ for 5 minutes and aliquoted and stored at $-80^{\circ} \mathrm{C}$ (passage 1). For further expansion, $300 \mu \mathrm{L}$ of passage 1 virus including infected cells was used to resuspend a pellet of $10^{6}$ VeroE6 cells in suspension. After incubation for 30 minutes at $37^{\circ} \mathrm{C}$, the co-culture was transferred to a 6 -well plate with $2 \mathrm{~mL}$ of MEM-2\%FBS medium. The resulting supernatant was harvested after 48 hours and cleared by centrifugation as above and stored at 
$-80^{\circ} \mathrm{C}$ (passage 2). A final larger expansion step (passage 3 ) occurred by resuspending $2 \times 10^{7}$ VeroE6 cells in $500 \mu \mathrm{L}$ of diluted passage 2 virus (multiplicity of infection 0.05 ), incubating at $37^{\circ} \mathrm{C}$ for 48 hours, and clearing and storing the supernatant at $-80^{\circ} \mathrm{C}$ as above. Shorter expansions with equivalent titres were also achieved by infecting the VeroE6-TMPRSS2 cell line with the same MOI but harvesting within 24 hours post-infection. Sequence identity and integrity were confirmed for both passage 1 and passage 3 virus via whole-genome viral sequencing as described further below.

\section{Titration of primary nasopharyngeal swabs}

Viral eluates from primary specimens were thawed and sterile filtered as indicated above. Samples were then diluted in 96-well plates (3-fold series, in quadruplicate) and $40 \mu \mathrm{L}$ of each dilution were transferred to an equal volume of freshly plated HAT-24 cells seeded in 384-well plates (CLS3985, Corning) at $1.6 \times 10^{4}$ cells in DMEM-5\%FBS medium. After incubation at $37^{\circ} \mathrm{C}$ for 72-96 hours, whole wells were imaged by high-content brightfield microscopy, and images were binarily scored by experienced operators for cytopathic effects ( + or -$)$ to determine the viral endpoint titre (i.e., last dilution containing at least one ' + ' well).

\section{Whole genome viral sequencing}

Clinical respiratory specimens positive by diagnostic SARS-CoV-2 PCR were sequenced using an amplicon-based Illumina sequencing approach, as previously described ${ }^{10}$. Consensus SARS-CoV-2 genomes have been uploaded to GISAID (www.gisaid.org) and are publicly available as indicated in Supplementary Table S1.

\begin{tabular}{|c|c|c|c|}
\hline Variant & Lineage & Seq ID & GISAID ID \\
\hline Ancestral & A.2.2 & nCoV_2715 & hCoV-19/Australia/NSW4715/2021 \\
\hline D614G & B.1.319 & nCoV_2713 & hCoV-19/Australia/NSW4713/2021 \\
\hline Alpha & B.1.1.7 & RCoV0066 & hCoV-19/Australia/NSW-R0066/2021 \\
\hline Beta & B.1.351 & $\mathrm{nCoV} 2463$ & hCoV-19/Australia/NSW4463/2021 \\
\hline Gamma & P.1 & nCoV 2319 & hCoV-19/Australia/NSW4318/2021 \\
\hline Delta & B. 1.617 .2 & nCoV_2605 & hCoV-19/Australia/NSW4605/2021 \\
\hline Epsilon & B.1.429 & $\mathrm{nCoV} 1646$ & hCoV-19/Australia/NSW3646/2020 \\
\hline Zeta & P.2 & nCoV_2299 & hCoV-19/Australia/NSW1508/2021 \\
\hline Eta & B. 1.525 & $\mathrm{nCoV} 2444$ & hCoV-19/Australia/NSW-R0187/2021 \\
\hline Kappa & B.1.617.1 & RCoV0206 & hCoV-19/Australia/NSW-R0206/2021 \\
\hline Lambda & $\mathrm{C} 37$ & nCoV 2431 & hCoV-19/Australia/NSW4431/2021 \\
\hline $\mathrm{N} / \mathrm{A}$ & $\mathrm{C} 36$ & $\mathrm{nCoV} 1629$ & hCoV-19/Australia/NSW3629/2020 \\
\hline Omicron & B.1.1.529 & RCoV_1933 & hCoV-19/Australia/NSW-RPAH-1933/2021 \\
\hline
\end{tabular}


Supplementary Table S7. Genomic viral sequences for SARS-CoV-2 variants used in this study.

\section{Titration of expanded viral stocks}

For overnight titrations (20 hour format), HAT-24 cells were trypsinised, resuspended in DMEM-5\%FBS medium with Hoechst-33342 live nuclear dye (Invitrogen, R37605) at 5\% v/v, and seeded in 384-well plates (Corning, CLS3985) at $1.6 \times 10^{4}$ cells/well. For traditional titrations (72 hour format), HAT-24 or VeroE6 cells were seeded at $5 \times 10^{3}$ cells/well in DMEM5\%FBS or MEM-2\%FBS respectively and stained with Hoechst-33342 only after the 72 hour virus incubation. SARS-CoV-2 viral stocks were serially diluted (5-fold series) in cell culture medium in octuplicate and then $40 \mu \mathrm{L}$ of viral dilution were added to an equal volume of the freshly plated cells. Plates were incubated for either 20 hours (rapid overnight titration) or 72 hours (traditional method) before the entire plate area was imaged on an InCell Analyzer highcontent microscope (Cytiva, USA). Brightfield images were visually inspected by experienced operators and compared against negative and positive infection controls to score wells binarily for cytopathic effects (+ or -) to calculate TCID50-values according to the Spearman-Karber method ${ }^{17}$. Fluorescence images were processed with IN Carta analysis software (Cytiva) to obtain total nuclei counts per well. For calculation of LD50 values, cell counts were normalised so that $100 \%$ represents the average cell number for mock-infected controls and $0 \%$ for the

highest viral concentration tested. LD50 values were obtained with GraphPad Prism software using the non-linear regression for dose-response with variable slope and four parameters. Viral titration on VeroE6 cells was performed exactly as described above, except cells were seeded at $5 \times 10^{3}$ cells/well in MEM-2\%FBS medium and stained with Hoechst-33342 after 72 hour incubation.

\section{Rapid high-content SARS-CoV-2 microneutralisation assay with HAT-24 cells (overnight R-20 assay)}

HAT-24 cells were trypsinised, resuspended in DMEM-5\%FBS medium with Hoechst-33342 live nuclear dye (Invitrogen, R37605) at 5\% v/v, and seeded in 384-well plates (Corning, CLS3985) at $1.6 \times 10^{4}$ cells/well. Human sera or monoclonal antibodies were serially diluted (2-fold) in DMEM-5\%FBS and mixed in duplicate with an equal volume of SARS-CoV-2 virus solution at $2 x$ the median lethal dose (2xLD50), calculated as indicated above. After 1 hour of virus-serum coincubation at $37^{\circ} \mathrm{C}, 40 \mu \mathrm{L}$ were added to an equal volume of pre-plated cells. Cell plates were then incubated for 20 hours before direct imaging on an InCell Analyzer HS2500 high-content fluorescence microscopy system (Cytiva). Cellular nuclei counts were 
obtained with IN Carta automated image analysis software (Cytiva), and the percentage of virus neutralisation was calculated with the formula: $\% \mathrm{~N}=(\mathrm{D}-(1-\mathrm{Q})) \times 100 / \mathrm{D}$, where " $\mathrm{Q}$ " is a well's nuclei count divided by the average count for uninfected controls (defined as having $100 \%$ neutralisation) and $\mathrm{D}=1-\mathrm{Q}$ for the average count of positive infection controls (defined as having $0 \%$ neutralisation). For additional detail and the rationale behind this formula, see Supplementary Figure S8 in ${ }^{20}$. The cut-off for determining the neutralisation end-point titre of diluted serum samples was set to the last consecutive dilution reaching $\geq 50 \%$ neutralisation for the average of technical replicates. Unless otherwise specified, samples were tested at a starting dilution of 1:40.

\section{High-content SARS-CoV-2 microneutralization assay with VeroE6 cells}

Human sera or monoclonal antibodies were serially diluted (2-fold) in MEM-2\%FBS and mixed in duplicate with an equal volume of SARS-CoV-2 virus solution at $1.25 \times 10^{4}$ TCID50/mL. After 1 hour of virus-serum coincubation at $37^{\circ} \mathrm{C}, 40 \mu \mathrm{L}$ were added to an equal volume of freshly trypsinised VeroE6 cells pre-seeded in 384 -well plates at $5 \times 10^{3}$ cells/well in MEM-2\%FBS (final MOI = 0.05). Cells were then incubated for 72 hours and subsequently stained with Hoechst-33342 (Invitrogen, R37605) at a final concentration of 5\% v/v. Entire well surface areas were then imaged, nuclei enumerated, and \% neutralisation determined exactly as indicated above for the R-20 assay.

\section{Air-liquid interface cultures}

Culture and differentiation of pBEC at air-liquid interface (ALI) was performed according to previously described methods ${ }^{27}$. Briefly, cells were seeded at $2 \times 10^{5}$ cells in 12 well plate transwells (Corning, 3460) and initially grown submerged in ALI-initial media, comprised of; 50\% BEBM-50\% DMEM, containing; $0.1 \%$ hydrocortisone, $0.1 \%$ bovine insulin, $0.1 \%$ epinephrine, $0.1 \%$ transferrin, $0.4 \%$ bovine pituitary extract (all from Lonza SingleQuotes ${ }^{\mathrm{TM}}$, CC-3171), and ethanolamine (final concentration $80 \mu \mathrm{M}$ ), $\mathrm{MgCl}_{2}$ (final concentration 0.3 $\mathrm{mM}$ ), $\mathrm{MgSO}_{4}$ (final concentration $0.4 \mathrm{mM}$ ), bovine serum albumin (final concentration 0.5 $\mathrm{mg} / \mathrm{mL}$ ), amphotericin B (final concentration $250 \mu \mathrm{g} / \mathrm{mL}$ ), all-trans retinoic acid $(30 \mathrm{ng} / \mathrm{mL}$ ) and $2 \%$ penicillin-streptomycin with $10 \mathrm{ng} / \mathrm{mL}$ recombinant human epithelial growth factor (rhEGF). Upon reaching confluence in the trans-wells (approx. 3-5 days after seeding), the rhEGF concentration was lowered to $0.5 \mathrm{ng} / \mu \mathrm{L}$ and cells were allowed to differentiate at ALI, without media in the compartment. After 25-30 days of differentiation, the quality of culture 
was confirmed by microscope observation of ciliated epithelium as well as presence of mucus/mucus-producing cells.

\section{Infection of ALI-pBECs and virus outgrowth assay}

Prior to infection, cells were washed once with phosphate buffered saline (PBS). Cells were inoculated with SARS-CoV-2 variants at 0.1 MOI, diluted in BEBM minimal media (Lonza; $\mathrm{BEBM}+1 \%$ ITS, $0.5 \%$ linoleic acid, $2 \%$ penicillin-streptomycin and $1 \%$ fungizone), on the apical surface only. After 2 hours incubation at $37^{\circ} \mathrm{C}$, the inoculum was collected, and the apical surface was washed with $500 \mu \mathrm{L}$ PBS to remove unbound virus. pBECs were harvested at 3 days and 7 days post infection. Upon harvest, apical washes were collected by addition of $500 \mu \mathrm{L}$ PBS for 5 minutes. All samples were stored at $-80^{\circ} \mathrm{C}$ until further use. A virus outgrowth assay was performed in HAT-24 cells to quantify infectious virus in apical washes. Samples were serially diluted 10 -fold in culture media (DMEM+5\% FBS) with a starting dilution of 1 in 5 , and $40 \mu \mathrm{L}$ of each dilution were transferred to cells pre-seeded in 384-well plates at $5 \times 10^{3}$ cells/well in $40 \mu \mathrm{L}$. Each sample was run in quadruplicate over 8 dilutions. Wells were imaged by brightfield microscopy at 72 hours post-infection, using an InCell Analyzer high-content microscope (Cytiva, USA). Cells were binarily scored by visual examination for cytopathic effect (+ or - ) and TCID50/mL values were calculated via the Spearman-Karber method ${ }^{17}$.

\section{Statistical analysis}

Sigmoidal dose-response curves and IC50 values were obtained with GraphPad Prism software using the non-linear regression for inhibitor versus response with variable slope and four parameters. The non-parametric Spearman's coefficient of correlation ' $r$ ' was calculated with GraphPad Prism using a two-tailed analysis with a confidence interval of 95\%. To compare the immune-evasiveness of different viral variants, titre fold-reductions were calculated for each subject and variant by dividing the IC50 from the subject-matched ancestral virus control by the IC50 of each variant. To test for statistical significance, the mean fold-reduction IC50s for each variant were compared to that of the 'wildtype' virus control (B.1) using a non-parametric Friedman test with Dunn's multiple comparison test. To calculate differences in variant infectivity, PCR-Ct values from primary nasopharyngeal swabs were plotted against endpoint titres from R-20 assay in HAT-24 cells. Titres were log transformed and linear regression was used to model the relationship between $\mathrm{Ct}$ values and titres for each variant. Statistical significance was assessed by comparing the slopes and intercept values for each variant and 
linear regression coefficient and Spearman's correlation coefficient determined $(95 \%$ confidence interval). Similar analyses parameters were employed to explore the relationship between age and virus titres.

\section{Ethics statement}

All human serum samples were obtained with written informed consent from the participants (2020/ETH00964; 2020/ETH02068; 2019/ETH03336; 2021/ETH00180). Primary bronchial epithelial cells (pBEC) were provided by P. A. B. Wark (University of Newcastle), and originally obtained from one healthy non-smoking donor (73-year-old female) during bronchoscopy, with written informed consent. Experiment was conducted with approval from the University of Newcastle Safety Committee (Safety REF\# 25/2016 and R5/2017). All subjects underwent fibre-optic bronchoscopy in accordance with standard guideline ${ }^{42}$.

\section{$\underline{\text { Key Resources Table }}$}

\begin{tabular}{|c|c|c|}
\hline REAGENT or RESOURCE & SOURCE & IDENTIFIER \\
\hline \multicolumn{3}{|l|}{ Antibodies and Sera } \\
\hline AB-3467 (monoclonal antibody) & 21 & AB-3467 \\
\hline WHO international reference standard & NIBSC & NIBSC (20/136) \\
\hline CoVIg-19 Plasma-Alliance standard & CSL-Behring & NA \\
\hline Polyclonal human IgG from pooled Plasma donors & CSL-Behring & NA \\
\hline \multicolumn{3}{|l|}{ Bacterial and virus strains } \\
\hline MAX Efficiency ${ }^{\mathrm{TM}}$ Stbl2 ${ }^{\mathrm{TM}}$ Competent Cells & ThermoFisher Scientific & 10268019 \\
\hline SARS-CoV-2 isolates & See Table1 & See Table1 \\
\hline \multicolumn{3}{|l|}{ Chemicals and reagents } \\
\hline DMEM & Gibco (ThermoFisher) & 11995073 \\
\hline MEM & Sigma-Aldrich & M4655 \\
\hline BEBM & Lonza & CC-3171 \\
\hline
\end{tabular}




\begin{tabular}{|c|c|c|}
\hline BEGM Bullet Kit SingleQuotes $^{\mathrm{TM}}$ & Lonza & CC-3170 \\
\hline PBS & Gibco (ThermoFisher) & 14190144 \\
\hline Fetal Bovine Serum (FBS) & Sigma-Aldrich & $\begin{array}{c}\text { F423-500 } \\
\text { (Lot\# 17G477) }\end{array}$ \\
\hline Trypsin & Sigma-Aldrich & T4049 \\
\hline Restriction Enzymes (AgeI, BsrgI, EcoR1, XhoI) & New England BioLabs & $\begin{array}{c}\text { R3552S, R3575S, R3101S, } \\
\text { R0146S }\end{array}$ \\
\hline Hoechst 33342 (NucBlue ${ }^{\mathrm{TM}}$ Live ReadyProbes ${ }^{\mathrm{TM}}$ ) & ThermoFisher Scientific & R37605 \\
\hline Resazurin (AlamarBlue ${ }^{\mathrm{TM}}$ Cell Viability Reagent) & ThermoFisher Scientific & DAL1025 \\
\hline Polyethyleneimine & Polysciences & $9002-98-6$ \\
\hline Ethanolamine & Sigma-Aldrich & E0135 \\
\hline $\mathrm{MgCl}_{2}$ & Sigma-Aldrich & M8266 \\
\hline $\mathrm{MgSO}_{4}$ & Sigma-Aldrich & M2643 \\
\hline Bovine serum albumine (BSA) & Sigma-Aldrich & A1595 \\
\hline Amphotericin-B & Sigma-Aldrich & A2942 \\
\hline All-trans retinoic acid & Sigma-Aldrich & R2625 \\
\hline Penicillin-streptomycin & Gibco (ThermoFisher) & $15070-063$ \\
\hline Recombinant human epithelial growth factor (rhEGF) & Sigma-Aldrich & E9644 \\
\hline \multicolumn{3}{|l|}{ Experimental models: Cell lines } \\
\hline HEK293T & ThemoFisher Scientific & R70007 \\
\hline HEK293T-ACE2-TMPRSS2-Clone\#24 (HAT-24) & This study & HAT-24 \\
\hline VeroE6 & ATCC & CRL-1586 \\
\hline VeroE6-TMPRSS2 & CellBank Australia & JCRB1819 \\
\hline
\end{tabular}




\begin{tabular}{|c|c|c|}
\hline Primary bronchial epithelial cells (pBEC) & $\begin{array}{l}\text { P. A. B. Wark } \\
\text { (University of } \\
\text { Newcastle) }\end{array}$ & NA \\
\hline \multicolumn{3}{|l|}{ Oligonucleotides } \\
\hline $\begin{array}{l}\text { Primers for hACE2 amplification: } \\
\text { Forward: ATT ACC GGT CGC CAC CAT } \\
\text { GTC AAG CTC TTC CTG GCT } \\
\text { Reverse: } \\
\text { TATTGTACATTAAGCGGGCGCCACCTGGGAGG } \\
\text { T }\end{array}$ & $\begin{array}{c}\text { Integrated DNA } \\
\text { Technologies (IDT) }\end{array}$ & NA \\
\hline $\begin{array}{l}\text { Primers for TMPRSS2 amplification: } \\
\text { Forward: ATAACCGGTCGCCACCATGGCCCTCA } \\
\text { A } \\
\text { Reverse: TTACTTGTACATTAGCCATCCGCCC }\end{array}$ & $\begin{array}{c}\text { Integrated DNA } \\
\text { Technologies (IDT) }\end{array}$ & NA \\
\hline \multicolumn{3}{|l|}{ Recombinant DNA } \\
\hline ACE2-ORF & Addgene & 1786 \\
\hline TMPRSS2-ORF & $\begin{array}{c}\text { Integrated DNA } \\
\text { Technologies (IDT) }\end{array}$ & $\begin{array}{l}\text { Custom synthetic gene } \\
\text { encoding human TMPRSS2 } \\
\text { (UniProtKB - O15393) }\end{array}$ \\
\hline pRRLsinPPT.CMV.GFP.WPRE (plasmid) & 41 & NA \\
\hline pLVX-IRES-ZsGreen (plasmid) & Clontech & 632187 \\
\hline psPAX2 (plasmid) & $\begin{array}{l}\text { NIH HIV Reagent } \\
\text { Program }\end{array}$ & ARP-11348 \\
\hline pMD2.G (VSVG plasmid) & Addgene & 2259 \\
\hline \multicolumn{3}{|l|}{ Critical equipment } \\
\hline InCell Analyzer HS-2500 (high-content microscope) & Cytiva & NA \\
\hline CLARIOstar® Plus (microplate reader) & BMG Labtech & NA \\
\hline \multicolumn{3}{|l|}{ Software and algorithms } \\
\hline GraphPad Prism version 8.0.0 & GraphPad Software & NA \\
\hline IN Carta (Image Analysis Software) & Cytiva & NA \\
\hline
\end{tabular}


Other

\begin{tabular}{|l|c|c|}
\hline 384-well microplate (tissue culture treated) & Corning & CLS3985 \\
\hline U-bottom 96-well microplates (for serial dilutions) & Greiner Bio-One & 650180 \\
\hline Millipore Ultrafree-MC centrifugal filter $(0.22 \mu \mathrm{m})$ & Merck & UFC30GV0S \\
\hline $\begin{array}{l}\text { 12-well plate with } 12 \mathrm{~mm} \text { Transwell@ polyester } \\
\text { membrane }(10 \mu \mathrm{m} \text { thickness, } 0.4 \mu \mathrm{m} \text { pore size) }\end{array}$ & Corning & CC-3171 \\
\hline $\begin{array}{l}\text { BEGM Bullet Kit SingleQuotes } \\
\text { TM }\end{array}$ & Lonza & \\
\hline
\end{tabular}




\section{Supplementary Files}

This is a list of supplementary files associated with this preprint. Click to download.

- TablesS1S6.xlsx

- Supplementarymovie1.mov 